\title{
Rubble Pile Asteroids
}

\author{
Kevin J. Walsh \\ Southwest Research Institute \\ 1050 Walnut St. Suite 300 \\ Boulder, CO 80302 \\ kwalsh@,boulder.swri.edu
}

Keywords: Asteroids, Rubble Pile, Solar System Formation, Collisional Evolution

\begin{abstract}
The moniker "rubble pile" is typically applied to all solar system bodies $200 \mathrm{~m}<$ Diameter $<\sim 10 \mathrm{~km}$ - where in this size range there is an abundance of evidence that nearly every object is bound primarily by self-gravity with significant void space or bulk porosity between irregularly shaped constituent particles. The understanding of this population is derived from wide-ranging population studies of derived shape and spin, decades of observational studies in numerous wavelengths, evidence left behind from impacts on planets and moons and the in situ study of a few objects via spacecraft flyby or rendezvous. The internal structure, however, which is responsible for the name "rubble pile", is never directly observed, but belies a violent history. Many or most of the asteroids on near-Earth orbits, and the ones most accessible for rendezvous and in situ study, are likely byproducts of the continued collisional evolution of the Main Asteroid Belt.
\end{abstract}




\section{0 "Rubble Pile"}

There is no guarantee that a moniker, such as "rubble pile" will, once coined, hold up over time. Here, used as a term to qualitatively describe the bulk nature of small asteroids and possibly comets, the term dating 40 years is still seemingly perfect (Chapman 1978; Chapman et al. 1978; Davis et al. 1985; Weissman 1986). In fact, the images of near-Earth asteroid Itokawa, returned by JAXA's Hayabusa space mission, could not have more clearly shown what scientists had inferred for decades about small asteroids. This small world looked like a pile of rocks from someone's garden or a mountain scree field. There are rough and angular boulders and cobbles littering the surface, one oddly large boulder looking out of place, and a few "ponds" of finer grains (Figure 1; Fujiwara 2006).

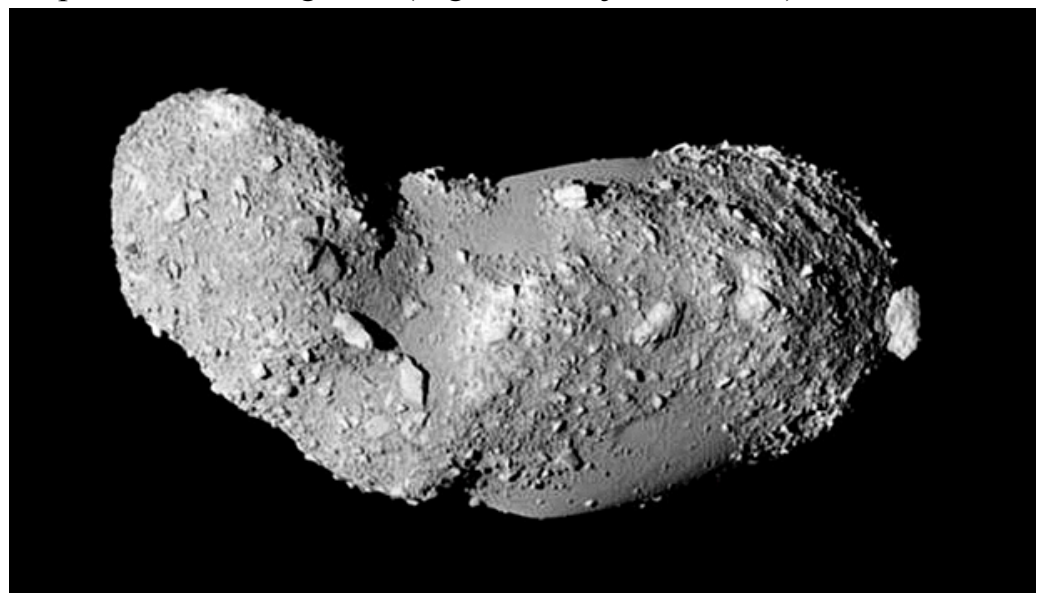

[Figure 1: A poster-child Rubble Pile, asteroid Itokawa. Image Credit \& Copyright: ISAS, JAXA]

The term "rubble pile" pre-dates the images of Itokawa by nearly three decades and was thus built on evidence compiled from astronomical observations, theoretical models and numerous researchers' intuition. The definition of a rubble pile was codified in a parameter space of tensile strength and bulk porosity, to be bodies with zero, or nearly zero, tensile strength and moderate bulk porosity, and therefore significant internal void space (Richardson et al. 2002). They were defined to be different than objects that were shattered, and thus strengthless and bound by gravity alone, but organized with minimal void space or porosity - something that could be referred to as a "shattered aggregate". While this community-wide effort to clarify the properties of small bodies in Richardson et al. (2002) specified a name of "gravitational aggregate" for low-strength and high porosity objects, the terminology of "rubble pile" remained in common use, and fully returned to prominence with the publication of the spectacular images of Itokawa in an article titled: "The Rubble-Pile Asteroid Itokawa as Observed by Hayabusa" (Fujiwara et al. 2006). Thus we embrace this name in this work, and aim to describe the essence of a "rubble pile": an unorganized collection of macroscopic particles (rubble) held together by their self gravity.

Some of the most specific data used to build the characterization of a rubble pile come from the study of near-Earth asteroids since members of this population regularly have close passages by the Earth enabling a wide array of ground-based observations - including radar ranging and doppler measurements that can be used to map an object's shape, spin and some surface properties. The near-Earth objects (NEOs or NEAs for near-Earth asteroids) weren't formed on their current orbits and will only be around 
this part of the solar system for an average of $\sim 10 \mathrm{Myr}$ before impacting a planet, the Sun or get ejected from the Solar System (Gladman et al. 2000). The population is transient and roughly in a steady-state, as asteroids that are removed due to collisions or ejection are replaced by new asteroids diffusing out of the Main Asteroid belt (Bottke et al. 2002b). Furthermore, the population contains all of the various spectral classes of asteroids found in the Main Belt, but they can't be directly linked back to a specific origin or orbit in the Main Belt. Significant modeling efforts provide only probabilistic accounting of their most likely pathways to reach their current orbits (Bottke et al. 2002b). Therefore we will largely skip a detailed discussion of the diverse taxonomy of asteroids found in the near-Earth and Main Belt populations, as they appear to have similar behavior in the data to be presented, despite having some different fundamental properties (amounts of volatiles, densities, spectra etc.: see DeMeo and Carry 2014). Similarly, most observed comets fit into the size range of typical rubble pile asteroids, and, by way of comet ShoemakerLevy 9 and 67P/Churyumov-Gerasimenko, provided substantial interest and evidence for rubble pile structures. However, while comets will be mentioned both in passing the bulk of the evidence for, and data on, rubble piles will be asteroid focused.

The concept of solar system bodies having limited strength dates back to Jeffreys (1947) who attempted to extend the work of Roche (1847) on disruption limits from fluid to solid bodies. The former speculated that solid bodies were not likely to tidally disrupt at any distance from a planet and that this was at odds with the formation of Saturn's rings or asteroids breaking up around Earth and Jupiter, whereas the latter had formulated orbital limits for fluid, or strengthless, bodies on orbits around planets. The discovery of Comet Ikeya-Seki in 1965, and its breakup during a close passage by the Sun, prompted further suggestions of strengthless small bodies (Öpik 1966). Similarly, as the orbital and size distributions of the Main Asteroid Belt came into view, collisional evolution via catastrophic disruption was found to likely be energetic enough to have created a large population of shattered leftovers with broken and loosely bound interiors (Chapman 1978). It was in this context that Chapman 1978 first used the term "rubble pile".

Population studies, specifically those that observed brightness variations over time to derive rotation rates (known as "lightcurves"), found a lack of very rapidly rotating bodies (Burns 1975; Pravec et al. 2000,2002). The critical spin limit, which no sizable (larger than $\sim 200 \mathrm{~m}$ ) asteroid exceeded, was $\sim 2.2$ hours (see Figure 2). This is similar to the rotation rate at which free particles could leave the surface of a spinning body of density similar to most meteorites. The absence of bodies with rotation rates faster than the observed limits argues that their internal structures do not allow it, but does not mandate it. This useful and highly suggestive dataset, with spin and shape as a combined constraint will be referred to later as a tool to estimate allowable shape and spin configurations relating to granular flow properties of known materials and to constrain their internal structures. 


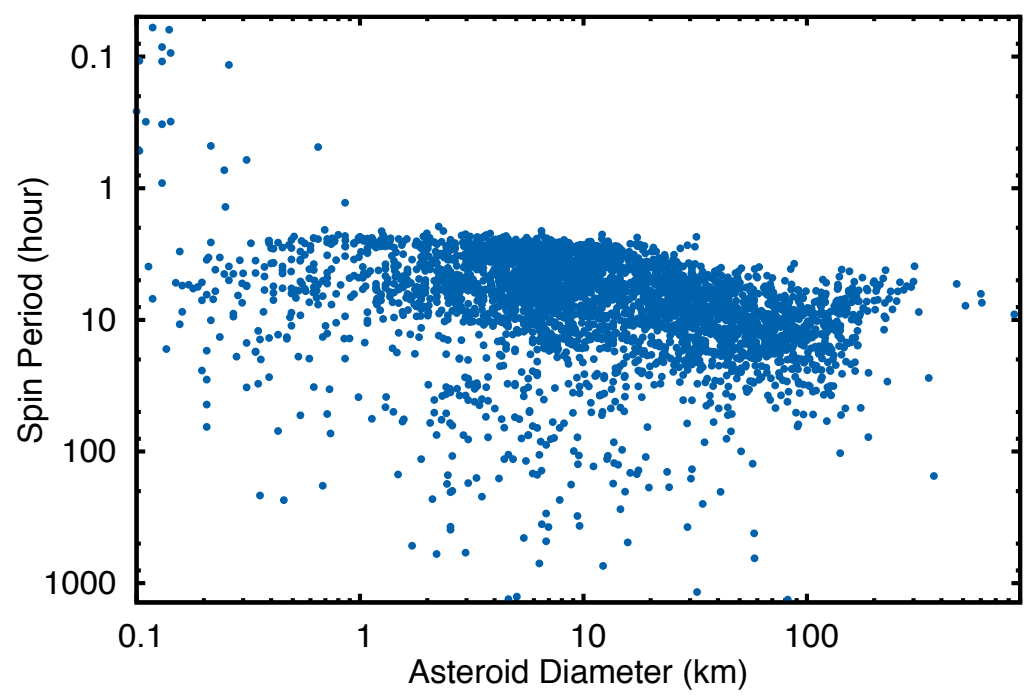

[Figure 2: The distribution of observed asteroid spin periods plotted as a function of their diameter $(\mathrm{km})$. This plot relies on the asteroid lightcurves recorded in the Planetary Data System archive (Harris et al. 2016) that have $U=3$ rating suggestive that they are very reliable. The two outliers, bodies with $\mathrm{D}>200 \mathrm{~m}$ and $\mathrm{P}<2 \mathrm{~h}$ are 2001 OE84 (Pravec et al. 2002) and $2001 \mathrm{VF} 2$ (Whitely et al. 2002).]

The surfaces of many planets and moons record information about the population of small bodies throughout the Solar System seen through the lens of crater formation. Crater chains in particular helped to drive the idea of fractured or rubble-ized interiors for small bodies that literally fall apart before impact, and helped to lead the way in understanding population-wide properties of small bodies. Crater chains, or "catenae", are linear features of individual impacts that can span tens of kilometers, and can be distinguished from possible local or endogenic sources (Figure 3). The Moon and the Galilean satellites have ideal and well-studied surfaces for these processes due to their proximity to a planet capable of tidally disrupting an asteroid or comet (where Saturn's low density make a violent disruption difficult Asphaug \& Benz 1996). The Moon has a few such crater chains (Melosh \& Whitaker 1994, Wichman \& Wood 1995) and Callisto and Ganymede have several on Jupiter-facing hemispheres (Schenk et al. 1996). The rate of expected events capable of making the observed crater chains pointed to a large population of strengthless bodies (Bottke \& Melosh 1996). 


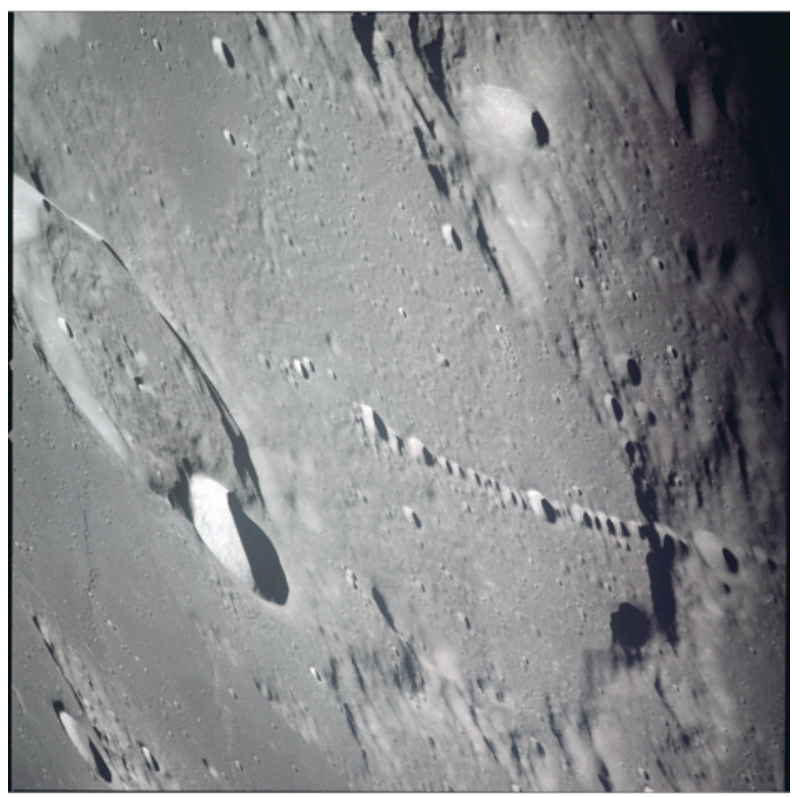

[Figure 3: The Catena Davy on the Moon, as captured in an image from Apollo 12. Photo number AS12-51-7485.]

A key difference between a rubble pile and an intact but broken "shattered aggregate" is the expectation of void space that should be a measureable bulk porosity. This is far from a trivial measurement to make, as knowledge of both mass and volume is required, which is a rare set of data to have for smaller solar system bodies. However, where the measurements exist, the occurrence of asteroids with densities significantly smaller than the grain densities for the most similar meteorites, has regularly pointed to moderate to high porosities for many asteroids, but almost without exception for smaller asteroids.

In 2004, the world finally saw a rubble pile up close, when JAXA's Hayabusa spacecraft visited the $\sim 500 \mathrm{~m}$ asteroid Itokawa, surveyed it, and even touched it and returned samples. Images of this asteroid have come to define what it means to be a rubble pile. Next up, to test and exercise these ideas and concepts are the upcoming space missions by both JAXA and NASA to visit, study and return samples from two more asteroids of similar size. Asteroids Ryugu and Bennu will be visited in the coming years, and both are much darker and spectrally distinct from Itokawa, belonging to the taxonomic groups that are suggestive of links to the more primordial and carbon-rich meteorites. All the data in this chapter suggest that both mission targets will be rubble piles and the community hopes to learn as much or more from them as was learned from Itokawa.

\subsection{Where do rubble piles come from?}


This is a two way street - big picture models of solar system evolution provide context important for understanding the character of small asteroids and comets, while properties of asteroids or groups of asteroids inform the big picture models of solar system formation and evolution. In this section solar system context is provided such that planetesimal formation models, asteroid belt collisional evolution and collisional physics conspire to suggest that there should be a population of rubble pile asteroids. However, the body of evidence about the nature of small asteroids has been building since the term "rubble pile" was coined in 1978 and predates much of the recent advances in Solar System evolution modeling.

\subsection{Planetesimal formation and solar system context}

The story of the Solar System's small bodies begins with one of the hardest problems in planetary science - planetesimal formation. Where, how and by what primary processes the first km-scale solid bodies in the Solar System are formed remains a generally open question (see recent review by Johansen et al. 2015). Several lines of evidence suggest that asteroids were "born big" (Morbidelli et al. 2009, Bottke et al. 2005, Johansen et al. 2015, Delbo et al. 2017), with sizes around one hundred kilometers, which is larger than what would today be considered to be the nominal size range for rubble pile asteroids.

The dynamics of the gaseous solar nebula will damp orbits and produce low relative velocities between dust grains and small particles $(<\mathrm{cm})$, which allows growth by sticking. But if particles were able to grow to sizes approaching one meter, aerodynamic drag from the gas disk would be maximized and result in significant loss of material due to rapid inward drift. Furthermore models of grain growth confront other barriers to growth whereby small mm-sized grains may simply bounce or fragment instead of sticking, effectively limiting the maximum size of simple growth at cm-sizes (Zsom et al. 2010, Wurm et al. 2005, Blum and Wurm 2008, Johansen et al. 2015). Therefore models rely on various gas-disk processes related to turbulence and instabilities to quickly collapse swarms of the $\mathrm{mm}$ - to $\mathrm{cm}$-sized particles that dominate the texture of most primitive meteorite samples and quickly form the much larger planetesimals (Cuzzi 2008, Johansen et al. 2007; Johansen et al. 2015).

The concept of a preferential initial minimum planetesimal size comes from both formation models and the analysis of the current asteroid size frequency distribution. An initial, characteristic, planetesimal size is fossilized in the current asteroid belt size distribution as a "bump" or a "knee" at D 100km (see Fig. 4; Bottke et al. 2005,2015; Morbidelli et al. 2009). This signature remains while some of the original population have suffered collisions, broken into smaller fragments and helped to populate the distribution at smaller sizes. This collisional evolution could drive an original belt entirely devoid of small objects (smaller than the characteristic D 100km) to its current state on billion-year timescales (Bottke et al. 2005). These studies suggest two important implications for everything smaller than this characteristic initial size:

1. They are not likely first generation, or original, members of the asteroid belt.

2. They likely formed as part of the collisional evolution/disruption of a larger body.

Constraints on both points can be solidified by understanding the collisional environment in the current asteroid belt. Collisional probabilities found in the current asteroid belt suggest that large collisions happen somewhat regularly (on Solar System timescales), and this is seemingly confirmed by the known suite of collisionally-formed asteroid families and the highly brecciated properties of many different types of meteorites (Bottke et al. 2005, 2015, Nesvorny et al 2015, DeMeo et al. 2015, Housen et 
al. 1982). Calculating expected timescales between catastrophic collisions for asteroids of a given size requires both the collisional probabilities and the physics that govern their shattering and dispersal.

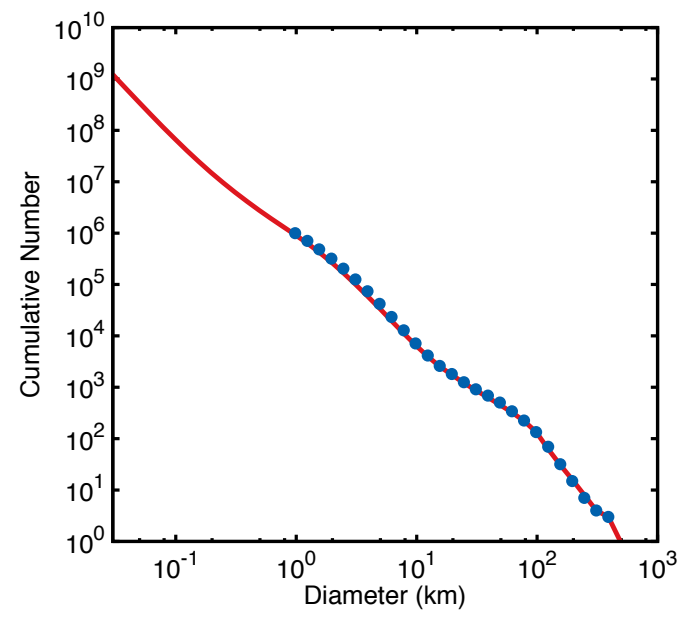

[Figure 4: The size frequency distribution of the Main Asteroid Belt. Of note is the structure of the curve around a few hundred meters and one hundred kilometers that is typically interpreted as relating to the size of the transition to strength-dominated bodies, and the size of the original population. Data from Bottke et al. 2005, 2015.]

\subsection{Collisional evolution - reaccumulating to form rubble piles}

Shattering and dispersal are essential steps in the larger concept of "collisional evolution" of asteroids, but so is the reaccumulation of their fragments. Shattering is something only applicable to a body with some bulk material strength where the required energy to shatter is typically measured with a value called the critical specific shattering energy, $Q^{*}$. This can be probed in terrestrial laboratories at $\mathrm{cm}-$ sizes and then at larger scales in hydrodynamic simulations (Nakamura et al. 1991, Benz \& Asphaug 1999). $\mathrm{Q}_{\mathrm{s}}{ }_{\mathrm{s}}$ specifies the projectile kinetic energy per mass required to shatter the target to the point where the largest fragment contains $50 \%$ of the system mass (Durda et al. 1998). The energy per mass required to shatter a body decreases as the targets get larger due to the internal flaws increasing with size (Housen \& Holsapple 1990; Holsapple 1994), and this trend holds up to the point that gravity becomes more important than strength.

Simply shattering bodies will not evolve the size distribution of the entire main asteroid belt - they must be shattered and dispersed to change the population statistics. The critical specific dispersing energy is $\mathrm{Q}_{\mathrm{d}}{ }_{\mathrm{d}}$, which is the energy required to leave a largest remaining remnant that is only $50 \%$ of the original target mass. This value increases with size as gravity grows more important, but at small sizes it is a bodies' strength that determines collision outcomes. The crossover point of strength and gravity regimes for unfractured basalt targets is estimated to be around D 300m (Asphaug et al. 2002) and is reflected in 
another bump/wave in the size frequency distribution of the asteroid belt that is expressed at sizes around $1 \mathrm{~km}$ (Bottke et al. 2005). This size is subject to many uncertainties regarding the possibly pre-fractured state of the target before its impact (Benavidez et al. 2012) and the averaged effect of different and potentially oblique impacts (Movshovitz et al. 2016) or pre-impact rotation (Ballouz et al. 2014). Furthermore, as is discussed below as part of the conclusions about internal structure deduced from shapes and spin, this size range is where bodies are found to be spinning more rapidly than what would be allowed for a body bound only by gravity - generally suggestive of some strictly monolithic strengthdominated bodies at and below this size.

When bodies are disrupted their debris not only disperses as individual strength-dominated monolithic fragments, but the dispersed fragments themselves are expected to reaccumulate into a series of new asteroids (Michel et al. 2001). This process has been constrained by the size distribution of reaccumulated fragments compared to observed collisional families (Michel et al. 2001, 2002 etc. Durda et al. 2004, Benavidez et al. 2015), and the formation of satellites (Durda et al. 2007). This points directly to the origin of rubble pile asteroids - by the reaccumulation of fragments following a catastrophic asteroid collision.

This reaccumulation process is difficult to model - as a huge number of fragments might be expected to reaccumulate following a giant impact. The actual impact and shockwave propagation through a target requires hydrodynamics simulations to track the shock propogation through a solid target, but once the target is shattered the dispersal and reaccumulation are governed by their gravitational attraction and relatively low-speed impacts. Therefore most efforts include a handoff from hydrodynamics to $\mathrm{N}$ body gravity particle codes (Michel et al. 2001,2002,2003,2004, Durda et al. 2004, 2007, Benavidez et al. 2015). The latter steps are numerically expensive and one of the many simplifications taken to keep simulations reasonably sized is to perfectly merge the particles reaccumulating into each individual fragment. While it is clear that all of the particles will form a new reaccumulated asteroid matching the size frequency distribution, no shape or spin information is retained to study the individual bodies in the newly built family of asteroids (see Figure 5; Michel and Richardson 2013, Walsh et al. 2017).
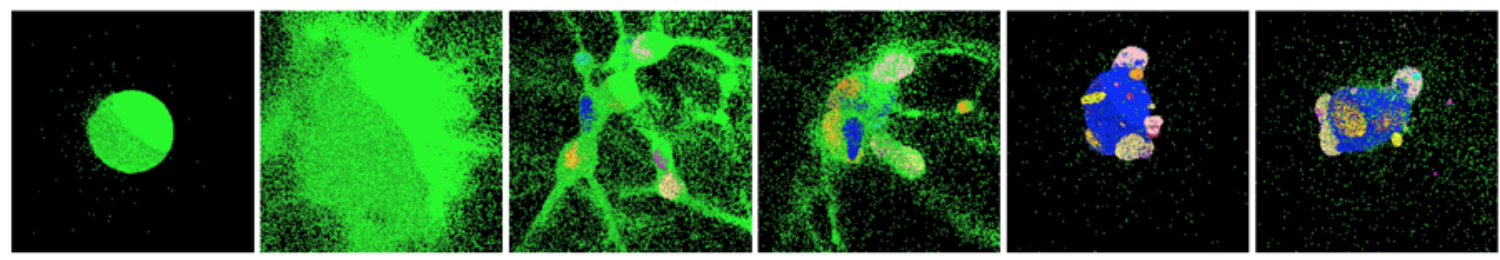

[Figure 5: The reaccumulation of fragments following an asteroid catastrophic collision, with a new numerical technique that keeps all particles in the system. Credit to Michel and Richardson 2013]

Collisional lifetimes, the expected time between catastrophic impacts for an asteroid of a given size, can be calculated with $\mathrm{Q}_{\mathrm{s}}{ }_{\mathrm{s}}$ and $\mathrm{Q}_{\mathrm{d}}{ }_{\mathrm{d}}$, and the orbit and size distribution of the asteroid belt (Bottke et al. 2005, 2015). The expected timescale between disruption events for a body increases with size in the gravity regime due to both increasing $\mathrm{Q}_{\mathrm{d}}{ }_{\mathrm{d}}$, due to increasing gravity and the decreasing number of available impactors massive enough to disrupt them. A $\sim 10 \mathrm{~km}$ body has a collisional lifetime similar to the age of the Solar System, and thus anything below this size should not have survived in its initial state (Bottke et al. 2005). This size sets the upper limit for what is typically expected to be a rubble pile as everything smaller than this is likely a reaccumulated remnant, and larger than this size could have survived roughly intact. 
Combined, expectations from formation and evolution models point to a size range between $\sim 200 \mathrm{~m}-10 \mathrm{~km}$ where a reaccumulated rubble pile structure should predominate. The lower end of the size

range for rubble piles is deduced from the $\mathrm{Q}^{*}{ }_{\mathrm{d}}$ curve and the transition from gravity dominated regimes to strength dominated regimes and is supported by the distribution of known spin rates to be $\sim 200 \mathrm{~m}$. The large end of this range is deduced from collisional lifetimes, modeling suggesting an initial characteristic size $\mathrm{D} \sim 100 \mathrm{~km}$, and is supported by the bump in the size distribution at these larger sizes. Spacecraft visits provide some insight as $\mathrm{D} \sim 34 \mathrm{~km}$ asteroid Eros is considered more likely a shattered, but intact, aggregate rather than a re-accumulated rubble pile based on its density and surface geology (Cheng et al. 2002), while $\sim 350 \mathrm{~m}$ Itokawa is clearly a rubble pile.

\subsection{Internal Structure}

By understanding the internal structure of rubble pile asteroids we aim to build constraints on their place in Solar System evolution, to understand how they have behaved and evolved over time, and to learn how to model them and interact with them now and in the future. Nearly all data on hand are astronomical - population data about shapes and spins and thermal and radar data about the surfaces. For a handful of objects there is actual resolved images of surfaces from flybys and rendezvous. These data constrain the modeling efforts that range from semi-analytic models of surface material flow to N-body models of gravitational bound particles with a variety of surface forces. Combined they build our picture of a rubble pile's internal structure.

The critical findings from the data and models suggest that while high porosity and a lack of tensile strength may be a defining property of a rubble pile, their shear strength and a resistance to reshaping is extremely important for defining the state and behavior of a rubble pile. The importance of shear strength is clarified in a simple example: while neither water nor sand on the beach has any tensile strength, the shear strength of sand is why we can walk on it and not water. Likewise, rubble piles show minimal resistance to disruption by a primarily tensile force such as tidal disruption, but if slowly spun to rapid rotation rates they may suffer only minimal re-shaping and can maintain spheroidal shapes. The attempt to characterize these properties, and the desire to model it, have driven much of the research in this field.

\subsection{Density, porosity and satellites}

A key trait for a rubble pile asteroid is the presence of void space, or macro-porosity, that is inferred by way of low density compared to analog meteorites (where any significant micro-porosity would seemingly be found in meteorite analogs). The first glimpse inside an asteroid is attained by way of measuring its density, but the bulk density calculation for an asteroid requires its mass and volume. The mass itself can only be measured in a few ways with the most widely used being orbital deflections, where asteroids can alter the orbits of other asteroids (see Carry et al. 2012). This can attain accuracies of a few percent for the largest asteroids but rapidly decreases in accuracy for smaller sizes. Spacecraft that have flybys with an asteroid can measure mass by way of the detected trajectory deflection or directly from the orbit, although this has been done for only a small handful of objects (Yeomans et al. 1997, 2000; Fujiwara et al. 2006, Patzold et al. 2011). For objects with both a shape measurement and a decades long 
measurement of its orbital drift rate by the thermal Yarkovsky effect, which is dependent on mass, a mass and density estimate can be made, as it was done for near-Earth asteroid Bennu (Chesley et al. 2014).

Meanwhile, the properties of a satellite's orbit can be used to attain the system mass and has the advantage of being equally effective for large and small asteroids, and with the population of $\sim 250$ known asteroids with satellites being spread throughout a wide range of dynamical groupings and asteroid sizes, this is the key technique for measuring the density of rubble pile asteroids. Depending on the discovery technique(s) the information come with some uncertainties, but in some cases they have rivaled anything returned from a space mission. The first discoveries of asteroid satellites around small asteroids were made with lightcurve observations - brightness fluctuation over time - where in the case of eclipses and occultations the existence of a secondary can be deduced (Pravec \& Hahn 1997, Mottola \& Lahulla 2000). Discoveries of satellites made with this technique can relay information on the primary mass and density with various assumptions about the relative density between the two components and the system geometry, where Pravec \& Hahn (1997) first reported a density of $1.7 \pm 0.4$ grams cm ${ }^{-3}$ for small nearEarth asteroid 1994 AW1.

Meanwhile, radar detection and characterization of binary systems has provided a wealth of data, directly providing constraints on orbits and absolute sizes of each component with one set of observations. Radar also provided valuable confirmation of the lightcurve discovery techniques after observing some of the same targets (Margot et al. 2002). Combined, the two techniques found that about $\sim 16 \%$ of all nearEarth asteroids have satellites (Margot et al. 2002), and have produced densities for 13 NEAs with diameter below $10 \mathrm{~km}$, estimating an average porosity of $35^{+38}{ }_{-35} \%$ (Carry et al. 2012). The object with the smallest uncertainties is $1999 \mathrm{KW} 4$ with porosity of $45 \% \pm 16$, which is comparable to the value derived by the spacecraft visit to asteroid Itokawa, $42 \% \pm 11$ (Ostro et al. 2006; Fujiwara et al. 2006).

Overall, these data point to significant void space and high porosity on average among NEAs in this small size range. For context, hexagonal closest packing of spheres is $\sim 74 \%$ efficient, leaving $26 \%$ void space, while irregularly, or jammed, packing of spheres is $\sim 63 \%$ efficient with $37 \%$ void space or porosity (Song et al. 2008). The average value found here is similar to the irregular packing, while a handful of NEAs have values that actually exceed $50 \%$, pointing to a potentially complex interior structure. It is notable that the large NEA Eros (D 34km), also visited by spacecraft, has an estimated porosity of $19 \% \pm 1$, which is part of the reasoning that drives its inclusion in the grouping of shattered aggregates rather than rubble piles (Carry et al. 2012; Cheng et al. 2002).

\subsection{Rotation rates and shapes constrain internal structure}

Seeking brightness fluctuation over time, lightcurve observations and data reduction have become increasingly automated over time resulting in a large database of objects ( 1757 with quality code $U=3$ with $200 \mathrm{~m}<$ Diameter $<10 \mathrm{~km}$ in the PDS archive v16; Harris et al. 2016). A single apparition of an asteroid can provide very precise data on an asteroid's rotation rate from the periodicity of the lightcurve but only weak constraints on its elongation from its amplitude due to the unknown geometry of the rotation axis relative to the observer. As the number of apparitions increase, the ambiguities about spin axis direction can be resolved and tighter constraints on shape and elongation can be derived and eventually shapes can be constrained using lightcurve inversion techniques (Durech et al. 2010, 2015).

Given the relative ease of establishing a rotation period for an object, first-order analyses of the entire population are typically made assuming the end-member case of a spherical body. This drives the 
common comparison seen in Figure 2, where the singular variable of spin rate is found to not exceed what would allow a surface particle to escape from the equator of a sphere. Long the anchor for presumptions of a strengthless interior this compelling relationship does not demand any particular interior structure but is a compelling argument for a lack of strength. There are only two well-characterized outliers, 2001 OE84 (Pravec et al. 2002) and 2001 VF2 (Whitely et al. 2002), although more have been suggested in a range of different surveys (Chang et al. 2015).

Lightcurves also provide information about axis ratios, where the lightcurve amplitude during one apparition provides constraints on the equatorial $b / a$ amplitudes, where $a, b, c$, are the long, intermediate and short principal axes respectively, and principal axis rotation around the $c$ axis is assumed. Multiple apparitions can constrain the spin pole direction and eventually all three axis lengths. After converting lightcurve amplitude from observed magnitude to an estimated axis ratio $(\Delta m \sim 2.5 \log (\mathrm{a} / \mathrm{b})$; note that other effects such as albedo variations can also affect brightness fluctuations so that this does not provide a perfect indicator of shape), there is an added dimension to the critical spin period comparison, where expected maximum spin rate will decrease with increasing elongation $\left(P_{\text {crit }} / 3.3 \mathrm{hrs}=\sqrt{ }((1+\Delta m) / \rho)\right)$. Figure 6 shows that with the critical spin period is still respected for the entire population of asteroids, for asteroids with $200 \mathrm{~m}<\mathrm{D}<10 \mathrm{~km}$.

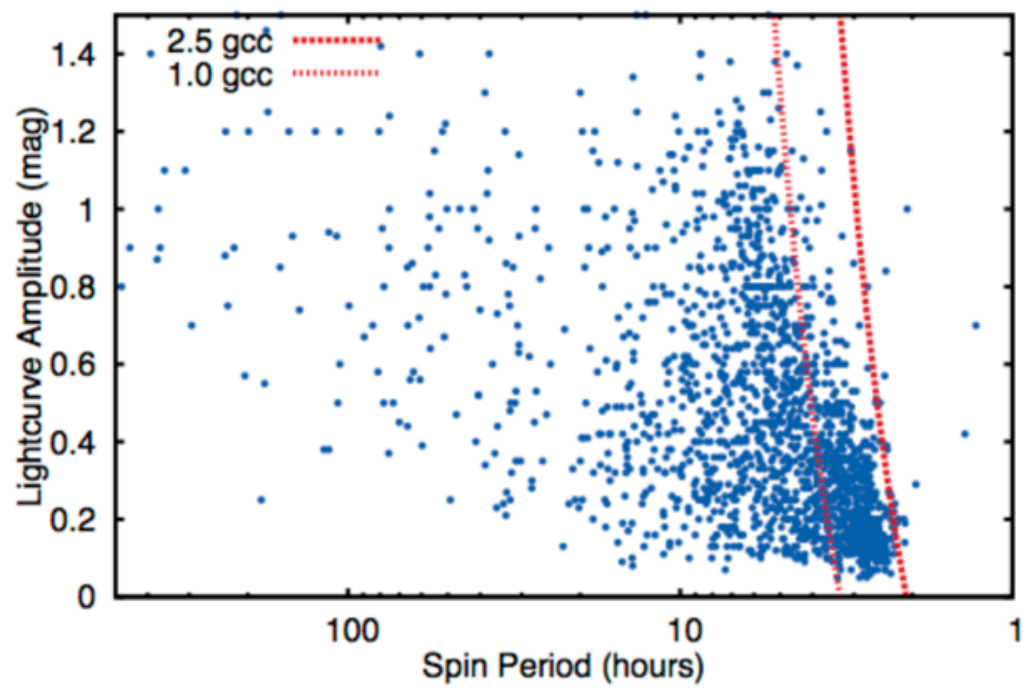

[Figure 6: Lightcurve derived amplitudes in magnitude plotted as a function of their spin periods (hours). The lines represent critical spin periods considering the lightcurve amplitude. Note that the maximum lightcurve amplitude is taken from the PDS archive, and geometry concerns only set this as a lower limit, which could result in moving some data points up (Harris et al. 2016).]

The simple fact that there are fast spinning spheroidal objects in this population demands that they are not behaving like fluids. A fluid, following the equilibria pathways described by Jacobi and MacLaurin, would immediately begin re-shaping after attaining non-zero angular momentum. Thus, the entire set of bodies in Figure 6, if they were acting as fluids should actually fall on a line, rather than fill a large space, and none should be in the lower right corner where rapidly spinning spheres are found. This all points the population having some amount of shear strength to be able to maintain non-fluid shapes while rotating (Farinella 1981, Holsapple 2001, Tanga et al. 2009, Richardson et al. 2005). This behavior relates quite naturally to a granular material, like gravel, where inter-particle friction and particle interlocking provides a shear strength that prevents reconfiguration of the materials. In simplest terms this 
is expressed in a materials "angle of friction" or "angle of repose", where it can withstand slopes up to a critical angle beyond which it flows.

Treating a rubble pile as a granular material opens up numerous modeling and characterization avenues. One such technique modeled rubble piles as cohesionless elastic-plastic solids, characterising the allowable shape and spin combinations, which can be outlined by an envelope in terms of axis ratios and rotation rate (Holsapple 2001, 2004). For the Mohr-Coloumb yield model the size of the allowable envelopes depends on the materials angle of friction, which is the primary input parameter (Holsapple $2001,2004)$. With this analysis the known data on asteroid spin states can be compared against allowable envelopes for a range of angle of friction (note that many authors have also deployed a Drucker-Prager model; Holsapple \& Michel 2006, Sharma et al. 2009, Rozitis et al. 2014). But, comparing the observations to the predicted envelopes is challenging due to widely varying densities, which are typically unknown and required for normalizing the observed rotation rates. Furthermore, a handful of outliers have been identified that absolutely must be bound by more than just gravity. Largely, this analysis finds that nearly all small asteroids could have their shape and spin configurations explained if their constituent material has an angle of friction similar to most known terrestrial materials, around $40 \mathrm{deg}$.

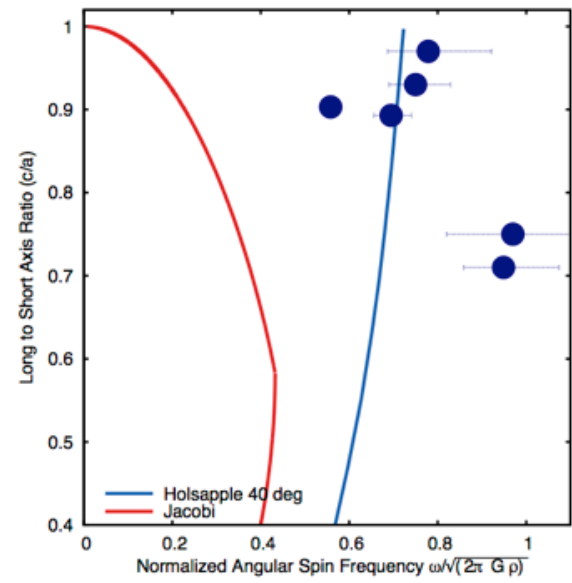

[Figure 7: Asteroids (from left to right), Bennu, 1999 KW4, 2001 SN263, 1994 CC, DP107, 1950 DA (Lauretta et al. 2014, Ostro et al. 2006, Rozitis et al. 2015, Naidu et al. 2015, Becker et al. 2015). Note that $1950 \mathrm{DA}$ was shown to require some cohesive strength to maintain its shape and spin configuration (Rozitis et al. 2014). All of the objects, except Bennu, have satellites, and 2001 SN263, 1994 CC are both triple systems.]

Individual bodies can be analyzed this way and compared directly against fluid equilibria and cohesionless spin limits (see Figure 7). A handful of near-Earth asteroids have very well-characterized shape models, densities and rotation rates (these are typically the primary of a binary system). They are typically found to have top-shapes or spheroidal shapes with equatorial ridges, which is discussed in depth below as they are suspected to have originated during an episode of spin rate increase. The comparison between their spin/shape configuration and these fiducials finds three of these six objects close to the 40 deg friction line of Holsapple (2001), two are well-beyond this limit, and Bennu is safely inside the 40 deg friction envelope. Of the two that are beyond the 40 deg friction limit, one is $1950 \mathrm{DA}$ which has been specifically modeled to have some cohesive strength ( $\sim 62 \mathrm{~Pa}$; see Rozitis et al. 2014). The minimal cohesion necessary to explain 1950 DA being so far beyond the allowed friction limits shows the 
difficulty with this type of analysis - even miniscule amounts of cohesion by terrestrial standards can quickly allow spin rates well beyond the limits allowed by simply friction and gravity.

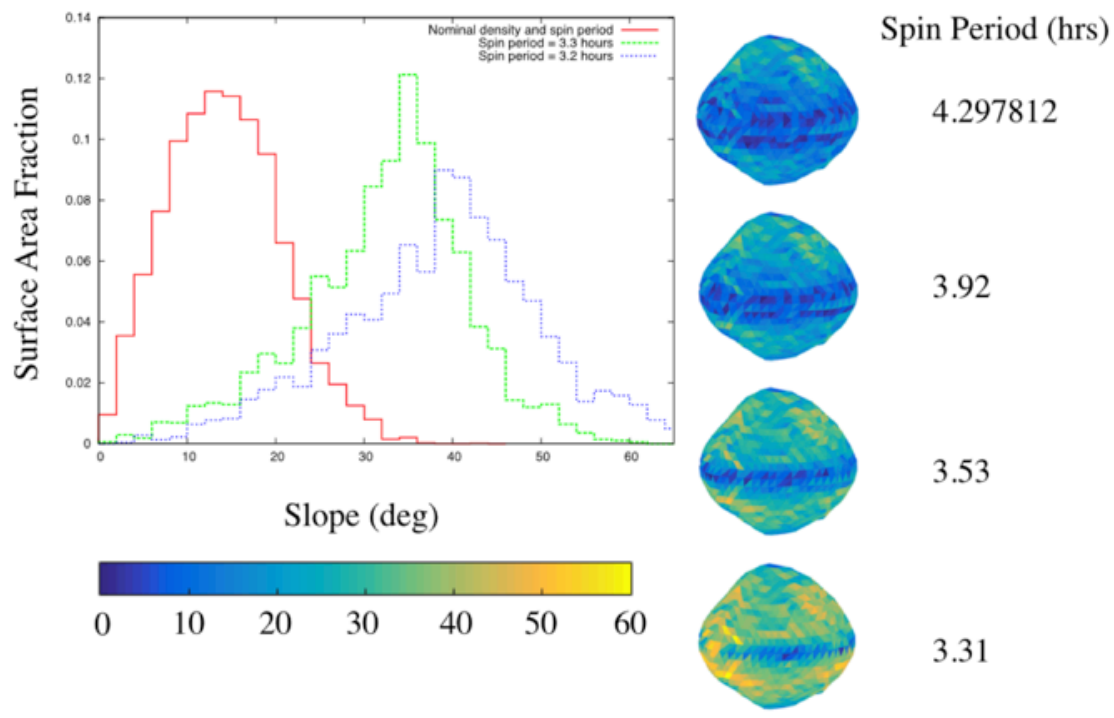

[Figure 8: The slope distribution across the surface of the asteroid Bennu at a range of spin states, where histograms show the fraction of the surface facets at a given slope for different spin rates of the asteroid. The slope distribution at the different spin rates is visualized in the images to the right following the colorscale on the bottom. Plot taken from Scheeres et al. (2016).]

On the other end of this group is the asteroid Bennu, which has the same top-shape found for all other objects in this group but is not near an extreme spin rate (see Figure 8). Here, if assumptions about the material properties are made - that they are similar to the other objects - then the distribution of surface slopes across its surface can be tracked using its spin rate as a free variable. In order to match slope distributions found on other rapid rotators, with peaks between $30-40$ deg., a spin rate of $3.3 \mathrm{hr}$ is needed, which is significantly faster than the current $\sim 4.3 \mathrm{hr}$ rotation rate. As will be discussed below, there are multiple mechanisms that can change spin states or shapes of near-Earth asteroids of this size, so this analysis of Bennu suggests a past history of more rapid spin and re-shaping.

\subsection{Odd shapes and how to reshape a rubble pile}

While the shape and spin configurations of rubble piles provides insight on their material properties they can also point to evolutionary mechanisms that have helped shape the population. These small bodies are subject to significant forcing due to interactions with planets (NEAs) and solar radiation providing valuable laboratories to understand them better. One of the most distinct features observed among small near-Earth asteroids are the spheroidal bodies with top-shapes or equatorial ridges. This particular shape is not trivial to determine - a lightcurve observation would witness only minimal brightness fluctuation owing to the nearly circular equator at all apparitions. Here the capabilities of delay- 
doppler imaging via radar has been able to interpret these shapes (Ostro et al. 2006). The width of the doppler returns track surface area at different rotation rates about a spin axis. The delay imaging follows the timing of returns and provides topography relative to the sub-earth point on the surface. Thus in the delay imaging the topography of the ridge is different than what would be returned from a perfect sphere. This shape is commonly found among the population of rapidly spinning asteroids that have satellites, suggesting that this is an end-state of a spin-up and binary formation process (Walsh et al. 2008, Harris et al. 2009, Scheeres et al. 2016), but is also found around at least one non-binary with moderate spin Bennu the target of NASA's OSIRIS-REx mission (Lauretta et al. 2015,2017).

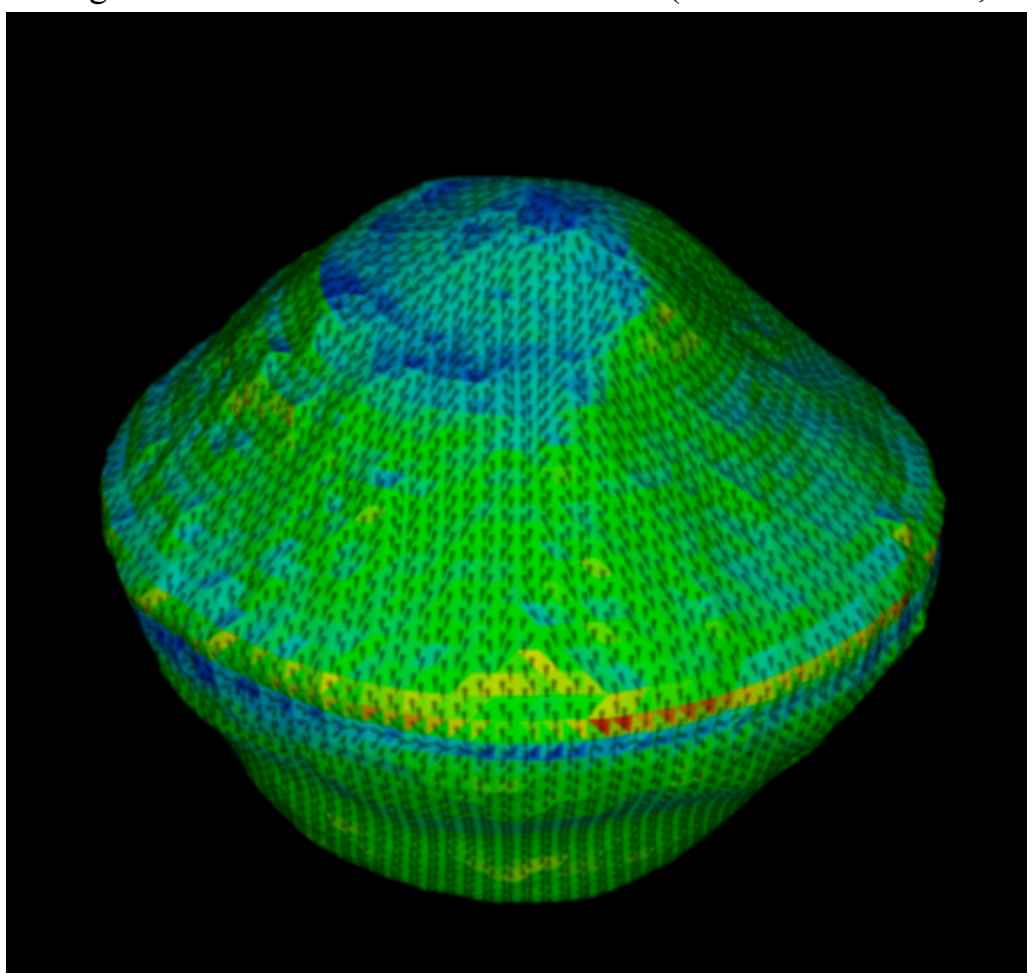

[Figure 9: Near-Earth asteroid $1999 \mathrm{KW}$, is found to have average surface slopes of 28 deg and maximums of $70 \mathrm{deg}$. It has a rapid rotation rate very near critical, and a satellite in a close and nearly circular orbit (Ostro et al. 2006, Scheeres et al. 2006). The colorscale shows slopes (blue is low and red is high, with the mid-latitude greens being $\sim 30 \mathrm{deg}$ ) and the arrows show the steepest descent.]

The other end of the spectrum are highly elongated, bifurcated and contact binary asteroids (Benner et al. 2015). Benner et al. 2006 estimated that $\sim 10 \%$ of NEAs $>200 \mathrm{~m}$ are contact binaries, following a strict definition for contact binary, where two lobes and bi-model mass distribution is required. Note that this definition would exclude objects such as Itokawa and Toutatis that have a shape suggestive of multiple components, but at a higher mass ratio between components (Fujiwara et al. 2006, Zhu et al. 2014). Using a relaxed definition that allows mass ratios between components as low as 4:1, which includes Itokawa and Toutatis, the fraction of the NEA population grows to $\sim 14 \%$ (Taylor et al. 2012). 


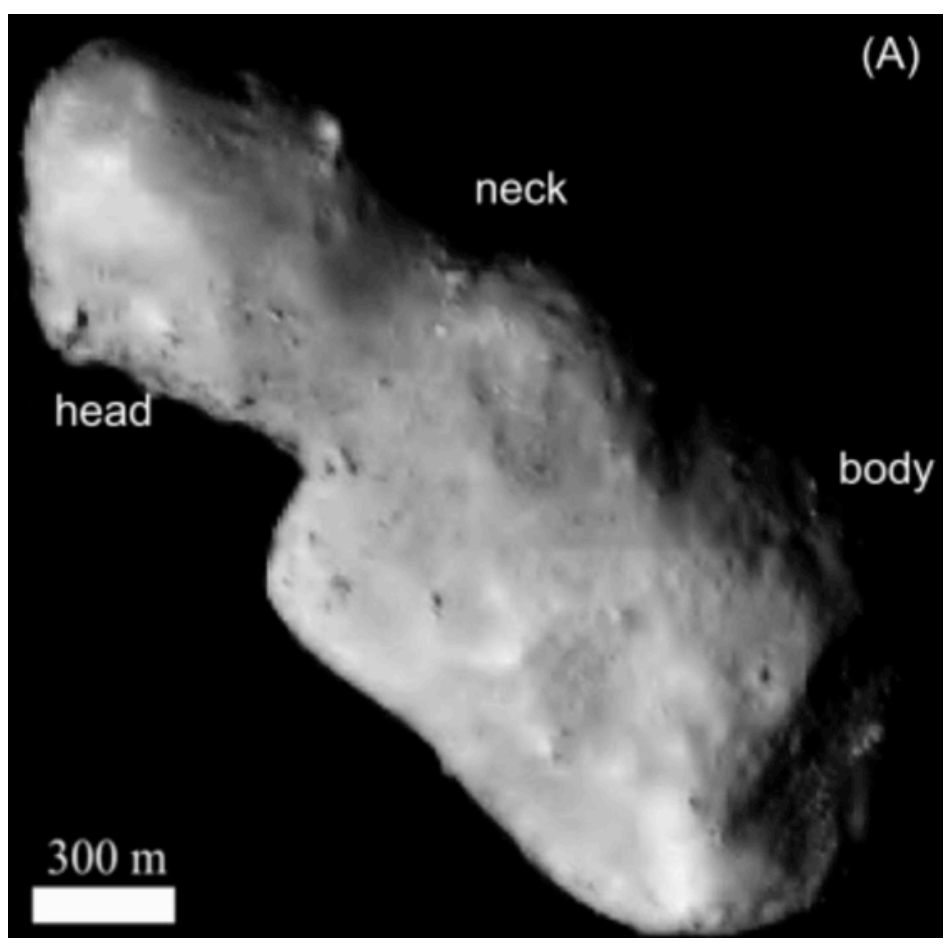

[Figure 10: The asteroid Toutatis is a km-scale NEA that shows a bifurcated shape. It was visited by the Chang'E-2 spacecraft (Zhu et al. 2014).]

The first effective dynamic models of the full-body behavior of rubble piles were motivated by the disruption of comet Shoemaker-Levy 9 (SL9) in 1992. Spectacular observations showed tens of fragments of the comet strung out along its orbit (Weaver et al. 1994), where the orbit traced backwards to a closest passage by Jupiter at only 1.31 Jovian radii (Yeomans and Chodas 1994). Such a close passage was likely within the Roche Limit, which is a classical disruption limit used widely to interpret close tidal interactions. However, the breakup was interpreted to have been quite violent given the large number of coherent and similar-sized fragments that were observed with no particular dominant member (Weaver et al. 1994). This event showed the inadequacies of simple disruption limit calculations such as the Roche Limit, which itself is built on assumptions of fluid equilibria, and instead demanded calculations and models more conscious of the interior dynamics of a body constructed of irregular shaped, and gravitationally bound, constituent pieces (Asphaug \& Benz 1994,1996; Solem \& Hills 1996, Richardson et al. 1998).

The first numerical models born from these studies were billiard ball, $N$-body, type models, those capable of modeling a gravitational aggregate, a body held together entirely by its self-gravity, but without some of the complicating dynamics introduced by surface penetrations and interactions of the constituent particles (Asphaug and Benz 1994,1996; Richardson et al. 1998,2000). Such a model was well-suited to capture the dynamics of tidal forces pulling an asteroid apart in a relatively impulsive event.

\subsubsection{Tidal Disruption}

The disruption limits and behavior of a simple rubble pile, in the absence of any tensile strength, only scale with density and not absolute size (Solem 1994), so models performed for SL9 can be applied 
to near-Earth Asteroids encountering the Earth and Venus or Trojan asteroids flying past Saturn and the outer planets (Asphaug \& Benz 1996). The starting point for these works is the classical Roche Limit, which derived the orbital distance at which a body would disrupt around a planet (Roche 1847). This limit is a valuable fiducial but was derived assuming a fluid body with a specific axis ratio and orientation relative to the orbit. Analytical modifications were made to account for realistic failure of brittle materials (Dobrovolskis 1990) and for a viscous body on a parabolic encounter, which substantially lowers the needed close approach distance for breakup (Sridhar and Tremaine 1992). These limits are simply functions of the relative density between the progenitor and the perturber or planet, but fundamentally describe at what distance something may disrupt, but not address how the magnitude or violence of the breakup nor the morphology of the resulting fragments nor can they easily account for even simplistic spin states or irregular shapes.

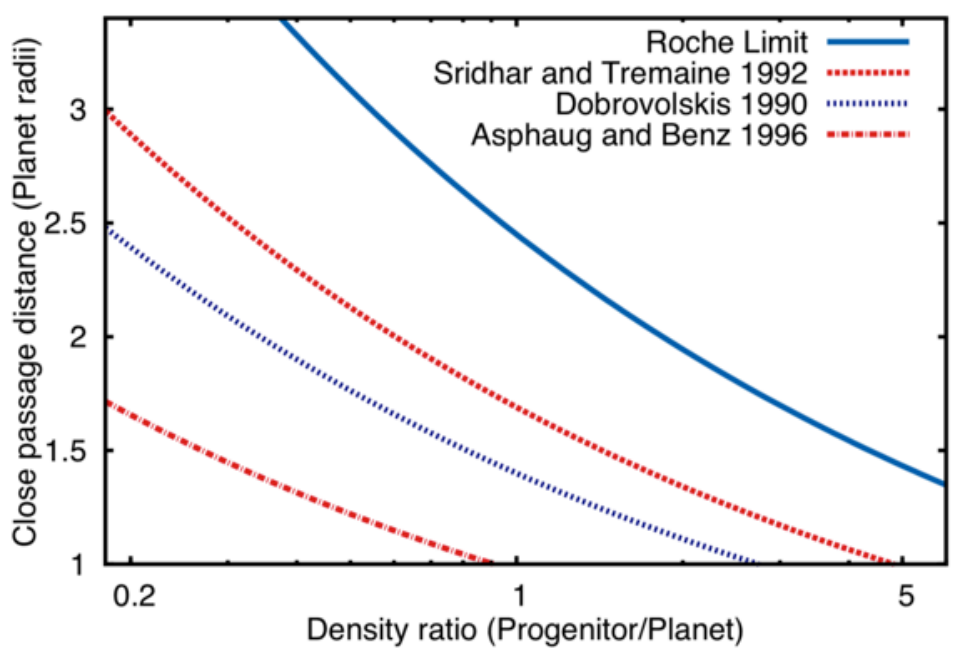

[Figure 11: Limits for tidal disruption plotted as the close approach distance to a planet in planetary radii as a function of the density ratio between the small body and the planet. The four calculation shown are the classical Roche Limit, the flyby limit from Sridhar and Tremaine (1992), the limit derived by Dobrovolski (1990), and the simulation outcomes from Asphaug and Benz (1996) for a SL9-type disruption.]

SL9 didn't just disrupt, it really disrupted. The imaging campaigns provided solid constraints for further modeling based on the fragment chain, and this was the launching point for the $N$-body approach to modeling rubble piles. The morphologic outcomes played a large role in understanding what the outcome should look like as a function of relative densities between progenitor and planet, which was constrained in a series of works to be quite low ( $\sim 0.6 \mathrm{grams} \mathrm{cm}^{-3}$; Asphaug \& Benz 1994,1996). Furthermore, these models allowed for a more detailed view of disruption, where the magnitude, or violence, of a disruption could be established and limits set for each. Richardson et al. (1998) built a basic descriptive taxonomy for levels of disruption based on mass loss:

- S-class, or catastrophic, disruption named after Shoemaker-Levy 9, where more than $50 \%$ of the progentior mass is lost,

- B-class disruption, where the largest remnant has between $50-90 \%$ of the total mass,

- M-class disruption that is mild where less than $10 \%$ of mass is lost. 
The SL9-type, or S-class, disruptions, require a density difference between the progenitor and the planet (see Figure 11). For the scaling found, an SL9 disurption would not be possible at Saturn for a comet with similarly low density as Saturn (Asphaug \& Benz 1996), whereas the terrestrial planets provide a large density difference than most small bodies and are good candidates to disrupt rubble piles.

The new modeling capabilities also permitted investigations into a wide range of spin states and shapes of the disrupting body (Asphaug \& Benz 1996, Richardson et al. 1998). Some important properties are degenerate during a tidal disruption - more rapid rotation in the prograde direction led to more violent breakups while retrograde rotation frustrated breakup. Thus rotation and close approach distance had some degeneracy, and the direction of the spin axis and the long-axis during close approach could both further complicate the outcomes (Richardson et al. 1998). Furthermore these efforts also clarified that even small amounts of tensile strength, provided by way of some sort of structural cohesive bonding, could seriously alter these outcomes and frustrate disruption (Asphaug \& Benz 1996, Holsapple \& Michel 2008).

Close tidal encounters have the capability to distort shapes, even in the case of no mass loss. The particular case of near-Earth asteroid (1620) Geographos stands out due to its highly elongated shape with irregular cusped ends and the close matches found in a simulation (see Figure 12; Bottke et al. 1999). How common this specific shape is throughout the population of bodies that can possibly encounter a terrestrial planet is not easily known, but the frequency of contact-binaries $(\sim 10 \%)$ and bifurcated shapes $(\sim 14 \%)$ have been quantified and are roughly as common as binary systems (Benner et al. 2006, Taylor et al. 2012). There are distinct modeling outcomes that produce shapes similar to Geographos, where a close approach of an ellipsoid with prograde rotation relative to its encounter experiences significant distortion and minimal spin rate increase, and ends up as a perfect doppelganger for Geographos (Bottke et al. 1999).

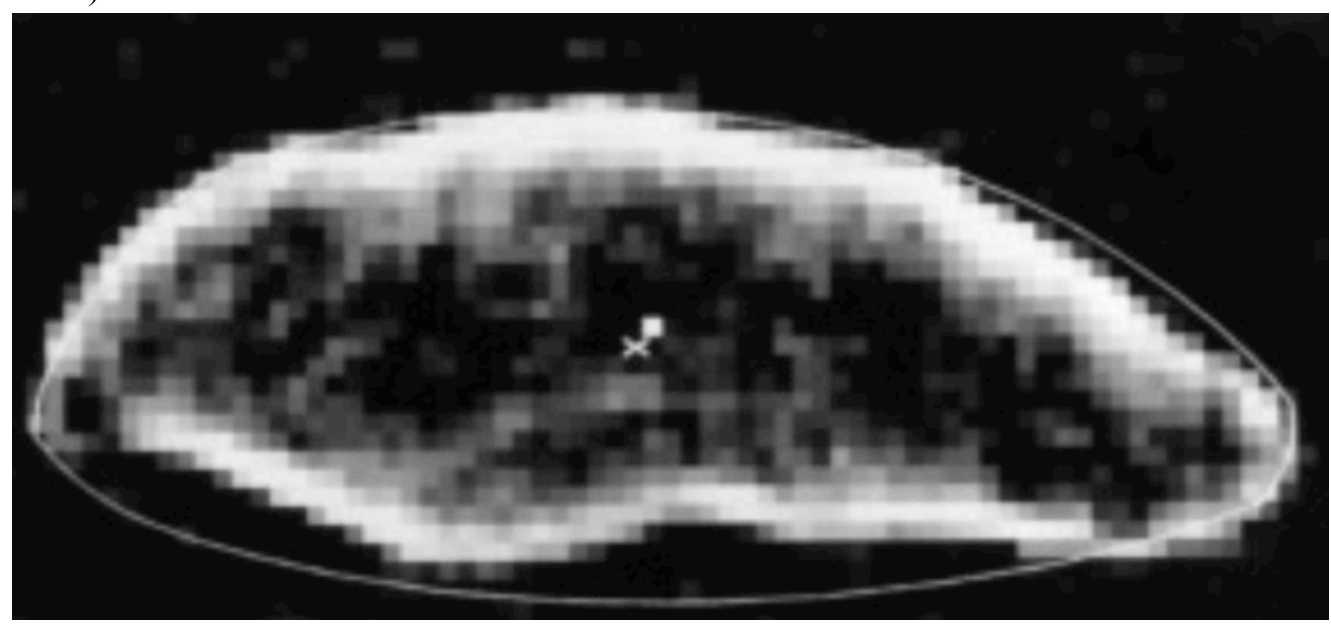

[Figure 12: Near-Earth asteroid Geographos was found to have a highly irregular shape, which was matched in N-body simulations of a tidal disruption (Bottke et al. 1999).]

Including more realistic surface interactions between the constituent particles, by way of a softsphere discrete element model (SSDEM; see Sanchez \& Scheeres 2011,2012; Schwartz et al. 2012; Zhang et al. 2017), actually generate more highly elongated shapes than found in the first generation of tidal disruption models (Walsh et al. 2017). In some cases a few pieces in the fragment train are bound due to the slight increase in frictional forces during disruption (a contrast to some previous models with minimal or no friction) and result in very low velocity collisions between fragments leading to some bifurcated 
shapes that resemble asteroids such as Itokawa, Toutatis etc., and could possibly account for some of the large population of contact binaries.

\subsubsection{Spin-up by the YORP effect}

The population of near-Earth asteroids with satellites makes up $\sim 16 \%$ of the entire near-Earth asteroid population (Margot et al. 2002, Richardson \& Walsh 2006, Margot et al. 2015, Walsh \& Jacobson 2015 ). The primaries are typically very fast rotators (periods $<4 \mathrm{hr}$ ), and the satellites are small and close (10-40\% the size of the primary and 2-4 primary radii away). While tidal disruption was shown to produce binary systems (Richardson et al. 1998, Walsh \& Richardson 2006), they were not great matches for these properties and lightcurve surveys found that a population similar to the near-Earth asteroids existed in the Main Asteroid Belt - far from any planet that was capable of tidally disrupting any asteroid (Walsh \& Richardson 2008, Pravec et al. 2006). Another mechanism was needed to explain this population and Bottke et al. (2002) proposed that a thermal affect similar to the Yarkovsky effect that changes orbits, known as the Yarkovsky-O'Keefe-Radzievskii-Paddack effect, or YORP-effect, might be capable of spinning asteroids to disruption (Rubincam 2000, Bottke et al. 2002).

[SIDEBAR: The YORP-effect relies on the asymmetries of an asteroid's shape to produce a torque around its spin axis when it reflects or re-emits solar radiation (Rubincam 2000, Bottke et al. 2006). The action of the YORP effect is directly observed in a handful of objects that are observed to be actively spinning up (Taylor et al. 2007, Kaasalainen et al. 2007) and long-term evolution is found in populations of asteroids that are driven into spin-orbit resonances in the Main Belt ("Slivan States" - Slivan et al. 2002; Vokrouhlický et al. 2003) and in the way that asteroid families drift apart over time (Vokrouhlický et al. 2006, Nesvorný et al. 2015). The YORP-effect can also alter obliquities, effectively pushing asteroids towards 0 or 180deg obliquities (Vokrouhlicky \& Čapek 2002; Čapek and Vokrouhlický 2004; Vokrouhlický et al. 2004). Whether or not a specific asteroid is likely to be spinning up or spinning down is nottrivial to calculate, even for a well-studied body with a known shape, and some studies suggest that even small scale surface artifacts like boulders or very small craters can change how a body responds (Statler 2009). ]

It's hard to overstate how small a given YORP torque would be on a $1 \mathrm{~km}$ asteroid. The time for a body of this size to halve or double its spin rate (YORP can spin down objects too) by this effect is on order $\sim 1$ million years (Bottke et al. 2006), which, while slow, leaves plenty of time during an average near-Earth asteroid lifetime ( $\sim 10 \mathrm{Myr}$ ) or the collisional lifetime of a $1 \mathrm{~km}$ main belt asteroid ( $\sim 500 \mathrm{Myr})$ for significant spin-state alteration. As implied by the spin and shape configurations of the entire population of asteroids there is an expectation of some shear strength acting in the form of a simple angle of friction - something to prevent these bodies from reverting back to fluid equilibria (Holsapple 2001; Richardson et al. 2005; Walsh et al. 2012). Spinning bodies up provides a way to slowly change angular momentum while maintaining the axis ratios of the bulk figure.

Models of granular flow can generate first-order estimates of bulk shape changes under increased rotation where the effective slope angle on a patch of the surface can dramatically change as the spin rate 
increases (as the spin approaches the critical $2.2 \mathrm{hr}$ limit the effective gravity at the equator goes to zero). As slopes pass the critical angles of repose failure of slopes is assumed and material is allowed to "flow" down to areas with lower potentials (Guibout and Scheeres, 2003; Walsh et al. 2008; Harris et al. 2009; Scheeres 2015). The bulk shape of 1999 KW4 is closely matched when the body has an angle of friction of $37^{\circ}$, which meets the expectations from the spin and shape distributions for all small asteroids (Harris et al. 2009). Failure is dominant in the mid-latitudes on spheriodal bodies, with material moving towards the equator, creating the observed equatorial bulge (Walsh et al. 2008; Harris et al., 2009; Scheeres 2015).

Similar $N$-body tools used for tidal disruption models were deployed to model how rubble piles would respond to the gradual torques provided by YORP spinup (Walsh et al. 2008,2012; Sanchez and Scheeres 2012,2016, Zhang et al. 2017). Models with simplistic or no surface forces mimic shear strength by hexagonal closest packing of similar sized spheres, or bi-modal distributions of spheres, finding that the spin-up process produces material flow towards the equator of spherical bodies that are qualitatively similar to the observed equatorial ridges observed (Walsh et al. 2008,2012). With better surface forces by way of the soft-sphere discrete element models (SSDEM) that allow modeling frictional material with a high ( $\sim 37 \mathrm{deg}$ ) angle of friction, the results become less straightforward as some failure modes appear internally in the evolving body, rather than just on the surface (Sánchez \& Scheeres 2012,2016, Zhang et al. 2017). While some of these outcomes can result in equatorial bulging, they often end up far more extreme than the sometimes subtle bulge seen in so many radar images. One possible solution is that a denser, or stronger, core of material is resisting internal deformation (Walsh et al. 2008, 2012; Hirabayashi et al. 2015; Sánchez and Scheeres 2016). In the first models the effective strength of the "core" was provided by a simple density increase for organized and closely packed spheres surrounded by unorganized and unpacked spheres (Walsh et al. 2008, 2012; Hirabayashi et al. 2015). However, the presence of a strong, or bound, core could come about by fine grains that have settled toward the center of a rubble pile and provide a cohesive binding (Sánchez \& Scheeres 2016) or simply by the reaccumulation process preferring to put the largest and most irregular blocks in the interior. Either route to providing a strong interior essentially pushes the failure towards surface shedding modes rather than internal failure.

The connection between the YORP spin-up process, its ability to generate shapes qualitatively similar to the ubiquitous top-shape and the observations that nearly all top shapes have satellites combine to tell a compelling story of rubble pile evolution. One of the oddities in the entire story of YORP spin-up and re-shaping is the asteroid Bennu. It has an equatorial ridge that is found among so many fast-spinning NEAs with satellites, yet its rotation rate is moderate and it has no known satellite. When it is visited and surveyed it will hopefully reveal geologic clues to point towards the origin of this notable shape.

\subsection{Tidal Dissipation}

There are many known rubble piles with satellites where the tidal interaction between the primary and the satellite could provide a means to probe their internal properties. The population of known satellites continues to grow, with more extreme systems continuing to be found (the number of triple systems increased in 2017), while other systems have been studied for decades long timescales (Scheirich et al. 2015). Nearly all known binaries among small, and likely rubble pile, asteroids have strikingly similar properties - with rapidly rotating primaries, close and small secondaries with orbital periods much longer than the rotation rate of the primary (see Margot et al. 2015, Walsh \& Jacobson 2015). The gravity of the secondary should raise a tidal bulge on the primary, but since the primary rotation is more rapid 
than the secondaries orbital period, this bulge is expected to lead the satellite. This bulge can then torque the satellite and evolve its orbit, while slowing the rotation of the primary. The timescale for these processes depend on the internal structure of both bodies and how they dissipate this tidal energy.

The application of any classical tidal evolution calculation could estimate the asteroid rigidity and dissipation factors (in similar terms as used for rocky planets) if the system timescale for tidal evolution is known (Margot et al. 2002). Near-Earth asteroids have relatively short lifetimes compared to Solar System history or typical Main Belt collisional timescales, averaging $\sim 10$ Myr. This average lifetime provides a rough timescale for evolution to their current orbital distance, leading to values of rigidity less than rocky planets (Margot et al. 2002). Beyond just the uncertainties in the evolution timescales critical to this calculation, the unique structure of rubble pile interiors could provide a dramatically different dissipation than typically assumed with tidal evolution models. Reformulated based on the consideration of energy dissipation at the few high-intensity points of contact within the body, the effective rigidity of the body decreases and tidal evolution can theoretically proceed much faster than for solid bodies (Goldreich \& Sari 2009).

However, confusing any estimation or calculation of a tidal timescale, is the continuing spin state evolution of the primary body caused by the YORP-effect, and its related effect that can evolve the orbit of the secondary, referred to as Binary-YORP, or BYORP (Ćuk \& Burns 2005; McMahon \& Scheeres 2010). In the same way that YORP provides a torque of a body using the asymmetry in its shape, BinaryYORP acts on the orbit of a satellite if it is tidally locked with its primary body, torqueing the entire system and changing the satellite's orbit. These two effects can operate simultaneously as tidal evolution, and potentially on much more rapid timescales (Jacobson \& Scheeres 2011). The timescales are potentially so rapid that they could outpace formation mechanisms and lead to depletion of the binary population, which seems unlikely in light of the large fraction of the population having satellites. Thus, an alternative tidal formulation was devised that scales directly as the BYORP orbit evolution, so that the observed population would actually be in an equilibrium between tidal torques pushing the satellite outward and BYORP driving the satellite inward (Jacobson \& Scheeres 2011). The critical tidal parameters of tidal dissipation $Q$ and rigidity by way of the tidal Love number $k_{2}$, would scale as $Q / k_{2}=6 \times 10^{5}(R / 1 \mathrm{~km})$, whereas the prior rubble-pile tidal model had the scaling as $Q / k_{2} \geq 10^{7}(1 \mathrm{~km} / R)$. The ambiguities between the orbit and spin torques caused by tides and thermal effects may only be resolved through long timescale observations of binary systems or smoking-gun detections of the BYORP effect in action.

\subsection{Cohesive strength}

A well-known and glaring assumption in most early modeling of rubble pile dynamics was the lack of any tensile strength or physical bonding between individual constituent pieces. As pointed out by numerous authors tidal disruption models were both greatly simplified and scale in simple ways when this can be ignored, and when it was ignored models found utterly reasonable outcomes in terms of derived densities and morphologies for SL9 and also in terms of the expected rates of disruptions relative to the observed crater chains on the Galilean satellites (Schenk \& Melosh 1993). Furthermore, calculations show that incredibly small amounts of cohesive strength can dramatically change outcomes - where $\sim 3$ Pascals was the maximum allowed cohesion in the best fit models for SL9's disruption (Asphaug \& Benz 1994,1996, Holsapple \& Michel 2006). 
What would be the source of cohesion in a rubble pile? If the primary "grain" size is related to the 200-300m size regime where rapid spin rates start to be observed, then one could assume that this is a fundamental building block size (Walsh \& Richardson 2006). Simple dry cohesive bonding by way of Van Der Waals force increases with decreasing grain sizes and would have no affect on such large rocks. However, images of asteroid Itokawa and inferences from thermal inertia data find that surfaces of rubble piles are covered with a wide range of much smaller grain sizes (Fujiwara et al. 2006, Delbo et al. 2015). If fine grains are also abundant in the interior of rubble piles then they could act as a sort of concrete with which to bind the larger constituent pieces of the rubble pile (Sánchez \& Scheeres 2014). As the cohesion increases so do the spin limits for small asteroids in particular, with those $\sim \mathrm{km}$ and smaller potentially experiencing faster allowable spin rates - such that the spin limit (Figure 2) would show a gradual increase in allowed spin rate at a large size depending on the cohesion limits. For example, $3 \mathrm{kPa}$ of cohesion would permit $1 \mathrm{~km}$ bodies to rotate faster than a $1 \mathrm{hr}$ period - which is not observed. Rather $100 \mathrm{~Pa}$ provides a comfortable envelope around the current set of observations (Sánchez \& Scheeres 2014).

In order to make a strong case that a given body demands cohesion to explain its shape and spin requires accurate data on all of density, axis ratios and spin period. While mass is typically the hardest to acquire, it has also be backed out from the observed orbital drift of an asteroid due to the Yarkovsky effect when combined with estimates of the bodies' thermal inertia derived from thermal modeling of thermal emission (Chesley et al. 2014). This dataset has all of the information required to make this analysis, and Rozitis et al. (2014) found that for asteroid $1950 \mathrm{DA}$, its rapid spin rate of $2.1216 \mathrm{hr}$, density of $1.7 \pm 0.7$ grams $\mathrm{cm}^{-3}$ could not be explained with simply gravity and friction alone. The Drucker-Prager model for internal strength required derived $64_{-20}^{+12} \mathrm{~Pa}$ of cohesive strength required for it to maintain its shape. Finite element models attain similar required values of cohesion, 44-76 $\mathrm{Pa}$, and describe a possible failure mode on the interior of the body resulting in equatorial bulging from the inside-out, rather than from mass movement from higher latitudes (Hirabayashi et al. 2015). The body also has remarkable thermal properties, evidenced by remarkably low Thermal Inertia, $24^{+20}{ }_{-14} \mathrm{~J} \mathrm{~m}^{-2} \mathrm{~K}^{-1} \mathrm{~s}^{-0.5}$, that strongly suggesting a surface covering of very small grains (the relationship between thermal response and surface properties is discussed in the next Section). This agrees in broad strokes with the description of cohesive binding of rubble piles by combined action of the smallest grains (Sánchez \& Scheeres 2014), and with possible cohesion limits derived for a main belt comet that was observed just after rotational disruption (Hirabayashi et al. 2014).

\subsection{Surface properties}

What about the nature of a rubble pile can be studied or investigated by way of its surface? A handful of asteroids have been visited and studied up close, but not all of the surface geophysics is applicable to actual rubble piles and their high-porosity interiors. It is clear that even sub-km sized rubble piles have small particle surface covering - a "regolith" - that reveals numerous interesting geologic effects dominated by the local gravity (Murdoch et al. 2015). But, a rubble-pile itself consists of loose and unconsolidated particles and, by some definitions of regolith, could simply consist entirely of regolith-like material with no clear delineation between interior and surface materials. We adopt the definition of regolith as the "loose unconsolidated material that comprises the upper portions of the asteroid" (as defined by Robinson et al. 2002), which makes no real strict limitations on where the regolith turns into 
the subsurface or internals of a rubble pile. Fundamentally, the surface is what is observed astronomically, and what a spacecraft is interacting with, and that is what we seek to understand.

\subsection{Nature of a rubble pile's regolith}

The combined study of small asteroids' surfaces suggest that most are covered with some sort of relatively fine-grained material - called "regolith". This is not obvious, especially for small asteroids and comets, which were once assumed to simply be monolithic rocks with no surface covering. A regolith was inferred over time due to studies of brecciated meteorite textures, remote observations of asteroids and eventually spacecraft visits (Housen et al. 1982, Sullivan et al. 2002, Robinson et al. 2002, Fujiwara et al. 2006, Biele et al. 2015). The fundamental impediment for regolith generation and retention is the very low escape velocities of small asteroids $(\sim 1 \mathrm{~m} / \mathrm{s}$ for a $1 \mathrm{~km}$ asteroid and increasing roughly linearly with diameter). Here, to zeroth-order, the collision speeds are so high in the main asteroid belt (averaging around $5 \mathrm{~km} / \mathrm{s}$ ) compared to the relatively low asteroid escape velocities, that a large portion of the impact ejecta will simply escape the target asteroid and not contribute to its regolith (Chapman 1976, Housen et al. 1982). This logic led to the notion that deeper and finer regolith coverings should scale with size and be found on only the largest asteroids (Housen et al. 1982), and the accumulation of data on the thermal response of asteroid's regolith largely support this conclusion.

Spacecraft have now visited asteroids of many shapes and sizes, from the largest, Ceres, down to Itokawa, finding some sort of regolith on all of them. These visits have validated many of the groundbased techniques and inferences made, but also established that small rubble pile asteroids, despite having less fine-grained regolith than larger bodies, still have interesting and complex surface geology. Before in situ data is available for an asteroid significant remote sensing capabilities are available to constrain the nature of its surface.

\subsubsection{Thermal inertia and thermal fatigue}

Regolith with different size grains have different thermal properties where large boulders and rocks retain daytime heat and re-radiate that heat slowly during nighttime. Meanwhile very fine grains quickly release any stored heat and provide a significantly different surface temperature profile over time. Observations in the thermal infrared wavelengths (where the wavelength correspond to the thermal emission from the surface, which depends on temperature and therefore distance from the Sun) can provide the data to model this behavior and constrain numerous properties about the surface (Delbó et al. 2015).

The thermal inertia of a body reflects the material heat capacity and the thermal conduction into and out of the subsoil, or deeper layers of the regolith. As thermal inertia goes towards zero the surface temperature increasingly matches the incoming solar flux leading to peak surface temperatures at noon with near-zero surface temperature across the nightside surface. As thermal inertia increases, this diurnal surface temperature curve get smoothed out. Absolutely critical to this process is the huge difference in conduction between large solid rocks and very fine-grained powdery lunar regolith, which can differ by three orders of magnitude. Therefore thermal inertia measurements relative to the Moon provide insight into typical grain sizes on an asteroid's surface.

A simple thermal modeling technique used when there is minimal knowledge of an asteroid's shape or spin is the near-Earth Asteroid Thermal Model (NEATM) (Harris 1998). NEATM necessarily 
makes assumptions about an asteroid's shape (spherical) to estimate an asteroid's diameter and albedo (Harris 1998), and encodes information about thermal inertia and surface roughness in a "beaming parameter", that can sometimes be used for comparisons of large numbers of objects (Delbó et al. 2007). More direct measurements of thermal inertia require full thermophysical models that rely on shape models of the target body and knowledge of its spin state. With this data in hand calculations for each surface facet can be made resulting in much stricter constraints on thermal inertia and surface roughness across an asteroid (Delbó et al. 2015).

The measured values of thermal inertia for asteroids is found to be size dependent (see Figure 12), where the largest asteroids have values approaching that for the Moon $\left(\sim 50\right.$ in these SI units, $\left.\mathrm{J} \mathrm{m}^{-2} \mathrm{~s}^{-1 / 2} \mathrm{~K}^{-1}\right)$, rapidly decreasing at smaller sizes. Rubble pile Itokawa provides a valuable marker with a thermal inertia of $700 \pm 200 \mathrm{~J} \mathrm{~m}^{-2} \mathrm{~s}^{-1 / 2} \mathrm{~K}^{-1}$ (Mueller 2007, Müller et al. 2014), in line with the imagery showing alternatively very rocky and boulder dominated terrain across much of its surface. Furthermore, relying on the physical foundation of thermal inertia being built on conduction between touching grains formalisms for turning a measured thermal inertia value into a grain size, or average grain size, have been built and can be compared to these measured values (Gundlach and Blum 2013). The asteroid Itokawa demonstrates the challenges in interpreting these calculations, as the surface shows very wide variations and no singular average grain size can fully describe it - despite the calculation estimating $\sim 22 \mathrm{~mm}$. Using this to make a relative comparison, for the asteroid Bennu, with its lower thermal inertia $(310 \pm 70)$, suggests at the very least a surface with more smaller grains throughout (Emery et al. 2014).

The same diurnal temperature cycles that allow astronomers to probe an asteroid's thermal properties also stress the grains and rocks that are being heated and cooled many times a day for millions or billions of years. This regular and constant thermal cycling has been explored in the lab and extrapolated in models to suggest that it could fracture and break down boulders and rocks and play a role in forming the fine grains found on asteroid surfaces (Delbo et al. 2014). Compared to an impact generation process for regolith production, which depends on the relationship between impact ejecta speeds and the bodies size and escape speed, thermal fatigue and cracking is size independent. This process may also be related to the loss of small bodies that have low perihelion - a notable absence discovered in recent modeling work - that could be thermal fatigue working in very destructive ways at much higher temperatures (Granvik et al. 2016)

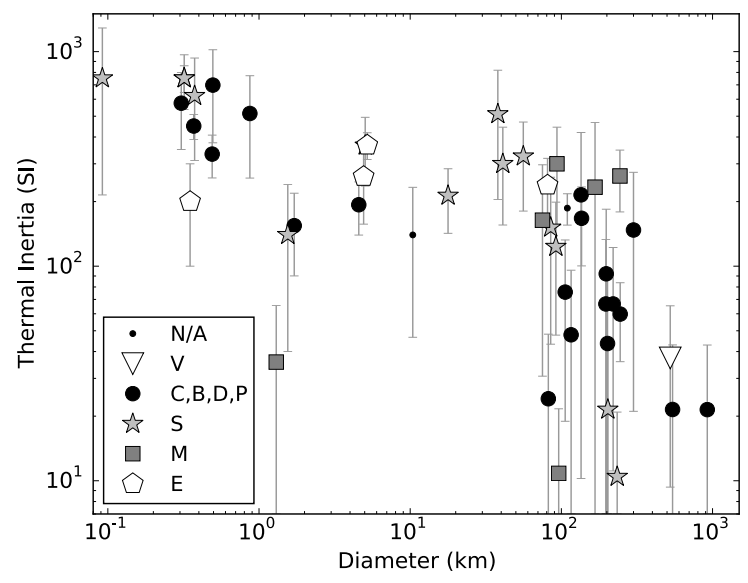

[Figure 13: Thermal inertia values for asteroids in units of $\mathrm{J} \mathrm{m}^{-2} \mathrm{~K}^{-1} \mathrm{~s}^{-0.5}$ plotted against their diameter in kilometers. The data are broken down into various asteroid taxonomy, but the trend 
stands for all for all them with higher thermal inertia at smaller sizes. Plot taken from Delbo et al. (2015).]

\subsubsection{Radar reflectivity and roughness}

Radar observations measure echo power from an object in both the time delay and doppler frequency, but they also include circular polarization recorded in terms of the opposite sense (OC) as transmitted and waves received in the same sense as transmitted (SC). In perfectly smooth scattering (relative to the lengthscale of the radar wavelength) the $\mathrm{OC}$ would dominate the return signal, giving a polarization ratio $\mathrm{SC} / \mathrm{OC}=0$. A surface that could completely randomize the reflected signal would approach $\mathrm{SC} / \mathrm{OC}=1$. For a given wavelength of observation the $\mathrm{SC}$ echos increase, and so does the SC/OC ratio, with increased surface roughness in length scales similar to that of the transmission wavelength (Ostro et al. 2002).

This method of observation has been "ground-truthed" by data from Itokawa (Ostro et al. 2004, Miyamoto et al. 2007). Itokawa was observed in both 3.5 and $12.6 \mathrm{~cm}$ wavelength radar, finding higher polarization at 3.5 than at $12.6 \mathrm{~cm}$, suggesting a changing surface texture at size scales between these two values (Ostro et al. 2004, Nolan et al. 2013). Meanwhile Bennu has a lower polarization ratios than Itokawa and similar values at both $3.5 \mathrm{~cm}$ and 12.6 , suggesting that Bennu has a larger population of few$\mathrm{cm}$ size grains relative to Itokawa and characteristic roughness scales smaller than $3.5 \mathrm{~cm}$ (Nolan et al. 2013). The ground-truth for Itokawa is not straightforward, as the surface is far from homogenous, with some very rocky and boulder-laden terrains and other ponds of very fine grains, but further ground-truths of these techniques should be obtained with the OSIRIS-REx visit to the NEA Bennu.

\subsection{Observed Surface Geology}

Itokawa is one of the best studied asteroids and the only rubble pile to be visited by spacecraft and therefore necessarily dominates discussion of surface geology on rubble pile asteroids. It showed that gravity is important even on very small asteroids, with gravitational slopes and potential lows being correlated with surface features. The absence of a large crater population or any large linear features combined with signs of regolith migration point to seismic shaking as an important energy source for surface modification (see Figure 1 \& 14: Miyamoto et al. 2007; Barnouin-Jha et al. 2008).

Itokawa had both rough and smoother terrains nominally defined by topography, with rough highlands and smooth lowlands (Saito et al. 2006, Miyamoto et al. 2007, Barnouin-Jha et al. 2008). The rougher highlands display surface roughness on scales of 2-4m, indicating a covering of meter-size boulders, and appear devoid of particles smaller than $1 \mathrm{~cm}$ (Barnouin-Jha et al. 2008, Miyamoto et al. 2007). Within the highlands there are a handful of smoother regions associated with localized depressions (Barnouin-Jha et al. 2008). The smoother lowlands are flat to sub-meter scales and essentially devoid of boulders (Barnouin-Jha et al. 2008), with some indications of possible crater-like depressions (Hirata et al. 2009). The roughness increases towards the edges of the smooth regions, which has led to the interpretation that the finer grains are migrating into the low-elevation areas and covering up the otherwise rocky terrain (Miyamoto et al. 2007, Barnouin-Jha et al. 2008). The boundary between the rough and 
smooth terrains shows signs of regolith movement and migration, with imbricated boulders aligned with local gravity slopes, pileups of small grains behind larger boulders and strong alignments of large irregularly shaped boulders (Miyamoto et al. 2007). The presence of rounded boulders similarly points to active geologic processes both moving and processing large surface particles (Marshall and Rizk 2015, Connolly et al. 2015.). The implied direction of these signs of regolith migration are in line with the local gravitational slope and demand a source of energy for their movement.

The small size and surface gravity of Itokawa, along with the global differences in terrain associated with potential lows and highs, point to seismic shaking as the key geologic driver on its surface (Miyamoto et al. 2007, Richardson et al. 2005). The combined action of small impacts result in effective gravel fluidization as impactors on cm-scales can impart enough energy to rival the surface escape speed regionally (Cheng et al. 2002). A question for this effect dominating on rubble piles is how widely the energy can be transported through a non-coherent and high-porosity interior. Miyamoto et al. (2007) suggested that seismic shaking could drive finer grains into the interior and subsequently change the energy attenuation of its non-monolithic rubble-pile interior. Both upcoming space missions to rubble piles, Hayabusa2 and OSIRIS-REx, will have the opportunity to look for further signs of these processes while also contrasting the surface geology between different taxonomic types of asteroids.

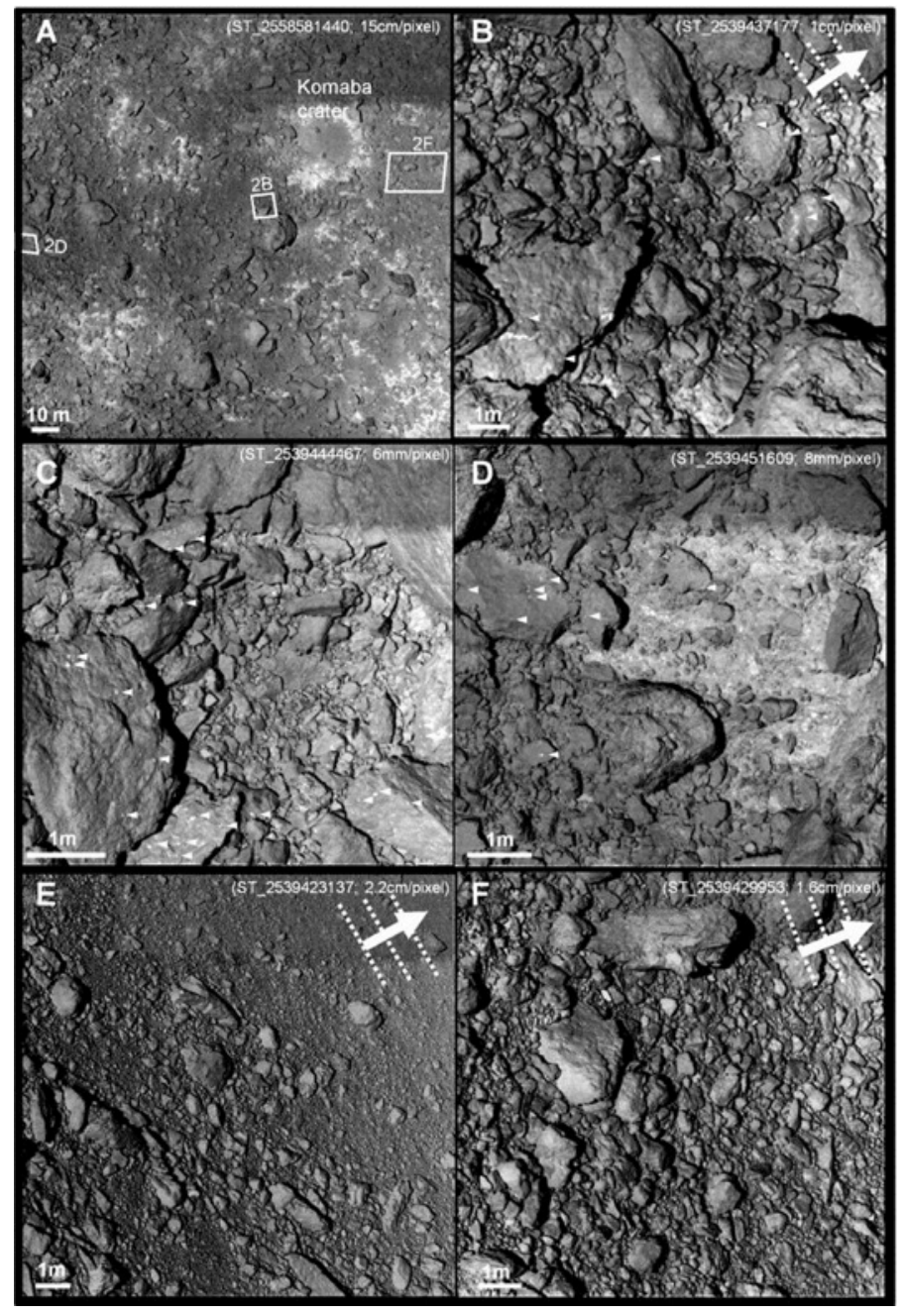


[Figure 14: The surface of Itokawa as imaged from JAXA's Hayabusa spacecraft taken directly from Miyamoto et al. (2007). A) This is the terrain around the Komaba crater at the resolution of $15 \mathrm{~cm} /$ pixel, and the outlined boxes show the field of view for panes B, D and F; B) Higher resolution image showing the organization of grains following the indicated lines and suggestive of flow in the direction of the arrow, C) $6 \mathrm{~mm} /$ pixel resolution shows large boulders piled on top of smaller regolith and the arrows indicate bright marking on some of the boulders, D) more rough terrain and strong albedo contrast, E) pile up of small grains is found on the uphill side of larger rocks in this terrain between rough highlands the smooth Muses $\mathrm{C}$ region and, F) in another area between smooth and rough terrain there are signs of imbrication.]

\subsection{Exploration}

Space exploration has advanced to the point where spacecraft regularly interact with the surfaces of planets (Mars and Venus), large moons (the Moon and Titan), asteroids (Eros and Itokawa) and also comets (9P/Tempel 1 and 67P/Churyumov-Gerasimenko). Of these Itokawa clearly fits the profile of being a rubble pile, partly due to its striking appearance. The interaction with the surface of Itokawa was brief, but the close-up imagery has provided a wealth of science. The upcoming space missions will do more and record more during their attempts to sample the surfaces of small rubble pile asteroids Ryugu (target of JAXA's Hayabusa2) and Bennu (target of NASA's OSIRIS-REx), and hopefully set the stage for even more ambitious efforts in the future. Both of these missions aim to return samples from the surface of their respective targets utilizing very different collection techniques.

While the combined knowledge of rubble pile asteroids can provide reference points for planning and provide tests for interpreting our observational and analysis techniques, it is also useful to ask what absolutely new things should be expected to come from these gigantic efforts. Both missions will survey their targets in an array of wavelengths, but both will also interact with their targets in completely new ways, which should provide important data about their surfaces and sub-surfaces.

\subsection{The Hayabusa2 cratering experiment}

JAXA's Hayabusa2 mission will undertake a spectacular experiment designed to reveal the subsurface of its target asteroid Ryugu. The spacecraft, launched in December, 2014, carries a kinetic impactor, the small carry-on impactor (SCI), which is designed to accelerate a $2 \mathrm{~kg}$ mass to $2 \mathrm{~km} / \mathrm{s}$ in order to make a sizeable crater on the surface of Ryugu (Saiki et al. 2017, Arakawa et al. 2017). Due to the violence of the explosion needed to accelerate such and impactor, and the possible debris launched during the formation of the crater on the surface of the asteroid, the main spacecraft will be moved out of view and a deployable camera will instead witness the event (Ishibashi et al. 2017).

The resulting crater is intended to reveal fresh material for later sampling, but also for remote sensing of this uncovered subsurface, unweathered, material to compare with the rest of the asteroid surface. The crater itself, its size and morphology, provides information about the near and subsurface of the target - whereby a singular large block being impacted provides strength-dominated cratering resulting in a crater up to $10 \mathrm{~m}$ across (Arakawa et al. 2017). Meanwhile an impact in a boulder field would still 
expend much of the energy into breaking individual rocks, but respond with a much smaller (as little as 1/10th the size) crater. Fluffy and porous layers on top of either of these pushes some of the response into the gravity-regime and keep the final crater relatively small as well. Meanwhile the deployed camera will view the ejecta curtain and should constrain the ejecta velocity distribution (Arakawa et al. 2017).

This impact should provide an instrument to test some of the large ideas around regolith migration on small bodies by actively driving a seismic shaking experiment. One of the primary inferred actions of seismic shaking has been the degredation of small craters and crater walls that could be observable (Richardson et al. 2005, Robinson et al. 2002, Michel et al. 2009), but the transmission of a seismic wave through the regolith layer (Yasui et al. 2015) and throughout the subsurface structure should provide some information on the internal structure of Ryugu.

\subsection{The OSIRIS-REx surface interaction}

Bennu, the target of NASA's OSIRIS-REx mission, is very well characterized with astronomincal techniques, with a high resolution shape model and surface roughness characterization from radar observations (Nolan et al. 2013), thermal inertia from thermal infrared observations (Emery et al. 2014), and a density determined from analysis of orbital change due to the Yarkovsky effect (Chesley et al. 2014). Much of the discussion above made comparisons between Bennu and Itokawa based on this data, typically pointing toward expectations of an asteroid with characteristic surface roughness at the $\mathrm{cm}$-scales and average grain sizes below cm-sizes (Emery et al. 2014).

The OSIRIS-REx surface interaction with its target Bennu relies on a new and novel sampling approach that minimizes contact time with the surface by utilizing a touch-and-go system (Lauretta et al. 2017). Following the survey of its target OSIRIS-REx will do a series of navigation maneuvers to move from a 1-km orbit, onto a trajectory to intercept the asteroid, another series of maneuvers to match the lateral motion of the target sample site due to the asteroid's rotation, and then finally contacting the surface at $10 \mathrm{~cm} / \mathrm{s}$ with minimal lateral velocity $( \pm 2 \mathrm{~cm} / \mathrm{s})$ (Lauretta et al. 2017). To combat any cohesive bonding or induration in the sampling area pyro valves will open and release high-purity nitrogen gas after contact with the surface is determined. The gas will also redirect the fluidized material into the sampling collector that is extended on a $2.8 \mathrm{~m}$ arm extended from the main spacecraft's body. The sampling head is an annulus, ringed by a screen to capture material that is flowing through the device driven by the released gas. The sampling head also has 24 contact pads made of stainless steel meant "grab" very small particles (Lauretta et al. 2017).

This sampling attempt is a controlled science experiment on its own, as the spacecraft will impact the surface of Bennu at $\sim 10 \mathrm{~cm} / \mathrm{s}$ with a known mass and at a known spot on the surface studied that has been surveyed in numerous wavelengths. While the returned sample will provide very tangible data on the material at the point of contact, the dynamics of the spacecraft during sample will probe the upper layers of the surface of Bennu. Spacecraft telemetry will record accelerations on the main spacecraft with high frequency so that the force imparted on the spacecraft during contact will be recorded. The spacecraft is dynamically separated from the asteroid's surface by a constant-force spring, and there is a maximum time allowed before the backaway burn will commence, so the total interaction is quite short, but while the spring will obscure some of the force imparted by the asteroid there will likely be a record of the initial contact and penetration (Lauretta et al. 2017). Force profiles during contact/impact can be used to characterize the material properties of the surface (Goldman \& Umbanhowar 2008) and can hopefully be 
used to detect the nature of the regolith - whether there are signs of stratification or layering at the surface, as inferred at the surface of comet 67P by the dynamics of the Philae lander (Schrapler et al. 2015, Biele et al. 2015).

\subsection{Conclusion}

Referring to small asteroids as "rubble piles" works. It describes all the lines of evidence and they support this generic moniker. From the thousands of collected lightcurves down to the rubbly and sizesorted surface of Itokawa, no data suggests that this is an unfair assessment of their qualitative nature. Furthermore, quantitative assessments of resistance to re-shaping, possible cohesive bonding, and comparisons against shear properties of standard terrestrial granular media really support them being just gravitational bound piles of rocks.

But, with just one of these objects studied up close, and two more in the sights of spacecraft, these ideas will all be tested again. Tripling the number of bodies for which surface geology has been studied and surface touched should enhance the data presented here and hopefully surprise the scientific community all over again. 


\section{SUMMARY POINTS:}

\section{FUTURE ISSUES:}

1. Detecting and understanding the Binary-YORP effect will be essential to understanding tidal evolution of rubble pile with satellites and learning more about their tidal dissipation.

2. Bennu has the ubiquitous top-shape suggestive of past spin up and reshaping, but it has a moderate spin rate and no satellite. Will it show signs of re-shaping, and if so, will it point towards internal failure or surface shedding and landslides?

3. JAXA's Hayabusa2 spacecraft will make a crater with its small carry-on impactor - will the crater formation process reveal much about the interior structure of Ryugu or will evidence of seismic shaking appear globally from the impact?

4. Are carbonaceous rubble pile similar, in bulk, to Itokawa or will the primitive asteroids Ryugu and Bennu, both set to be visited by spacecraft show different global properites?

5. What is the role of cohesion in rubble pile behavior and evolution? Will more novel studies constrain these values from astronomical data or will space mission visits shed more light by way of surface geology?

\section{ACKNOWLEDGEMENTS:}

K.J.W would like to acknowledge support and inspiration from NASA OSIRIS-REx asteroid sample return mission.

\section{REFERENCES:}

Arakawa M, Wada K, Saiki T, Kadono T, Takagi Y, et al. 2017. Scientific Objectives of Small Carry-on Impactor (SCI) and Deployable Camera 3 Digital (DCAM3-D): Observation of an Ejecta Curtain and a Crater Formed on the Surface of Ryugu by an Artificial High-Velocity Impact. Space Science Reviews 208:187-212

Asphaug E, Benz W. 1994. Density of Comet Shoemaker- Levy 9 deduced by modeling breakup of the parent "rubble pile." Nature, 370:120-124.

Asphaug E, Benz W. 1996. Size, density, and structure of Comet Shoemaker-Levy 9 inferred from the physics of tidal breakup. Icarus, 121:225-248

Asphaug E, Ostro S. J, Hudson R. S., Scheeres D. J., and Benz W. 1998. Disruption of kilometre-sized asteroids by energetic collisions. Nature, 393:437-440.

Asphaug E, Ryan E, Zuber M. 2002. Asteroid interiors. In Asteroids III (W. F. Bottke Jr. et al., eds.), this volume. Univ. of Arizona, Tucson.

Barnouin-Jha OS, Cheng AF, Mukai T., et al. 2008. Small-scale topography of 25143 Itokawa from the Hayabusa laser altimeter. Icarus 198:108-124 
Benavidez PG, Durda DD, Enke BL, Bottke WF, Nesvorný D, Richardson DC, Asphaug E. Merline WJ. 2012. A comparison between rubble-pile and monolithic targets in impact simulations: Application to asteroid satellites and family size distributions. Icarus 219(1):57-76

Benner LAM, Nolan MC, Ostro SJ, Giorgini JD, Pray DP, et al. 2006. Near-Earth Asteroid 2005 CR37: Radar images and photometry of a candidate contact binary. Icarus 182:474-481

Benner LAM, Busch MW, Giorgini JD, Taylor PA, Margot J-L. 2015. Radar Observations of Near-Earth and MainBelt Asteroids. in Asteroids IV 165-182

Benz W, Asphaug E. 1999 Catastrophic disruptions revisited. Icarus, 142:5-20.

Biele J, Ulamec S, Maibaum M, Roll R, Witte L, et al. 2015. The landing (s) of Philae and inferences about comet surface mechanical properties. Science 349(6247):aaa9816-1

Bottke WF Jr, Melosh HJ. 1996. The formation of asteroid satellites and doublet craters by planetary tidal forces. Nature 381:51-53

Bottke WF. Jr., Richardson DC, Michel P, Love SG. 1999. 1620 Geographos and 433 Eros: Shaped by planetary tides? Astron. J., 117:1921-1928.

Bottke WF. Jr., Vokrouhlický D, Rubincam DP, Broz M. 2002a. The effect of Yarkovsky thermal forces on the dynamical evolution of asteroids and meteoroids. In Asteroids III (W. F. Bottke Jr. et al., eds.), Univ. of Arizona, Tucson.

Bottke WF Jr, Morbidelli A, Jedicke R, Petit J-M, Levison HF, et al. 2002b. Debiased orbital and absolute magnitude distribution of the near-Earth objects. Icarus 156:399-433

Bottke WF, Durda DD, Nesvorny' D, Jedicke R, Morbidelli A, et al. 2005. The fossilized size distribution of the main asteroid belt. Icarus 175(1):111-40

Bottke WF, Vokrouhlický D, Rubincam DP, Nesvorný D. 2006. The Yarkovsky and YORP effects: Implications for asteroid dynamics. Annu. Rev. Earth Planet. Sci. 34:157-191

Bottke WF, Brož M, O’Brien DP, Bagatin AC, Morbidelli A, Marchi S. 2015. The collisional evolution of the main asteroid belt. Asteroids IV 701-724

Burns JA. 1975. The angular momenta of solar system bodies - Implications for asteroid strengths. Icarus 25:545554

Čapek D, Vokrouhlický D. 2004. The YORP effect with nite thermal conductivity. Icarus 172(2):526-536

Carry B. 2012. Density of asteroids. Planetary and Space Science 73(1):98-118

Chang CK, Ip W-H, Lin H-W, Cheng Y-C, Ngeow C-C, et al. 2015. Asteroid Spin-rate Study Using the Intermediate Palomar Transient Factory. The Astrophysical Journal Supplement Series 219:27-46 
Chapman CR. 1976. Asteroids as meteorite parent-bodies - The astronomical perspective. Geochimica et Cosmochimica Acta 40:701-719

Chapman CR. 1978. Asteroid collisions, craters, regolith, and lifetimes. In Asteroids: An Exploration Assessment, ed. D. Morrison and W. C. Wells, pp. 145-160. NASA Conf. Publ. 2053.

Chapman CR, Davis DR, Greenberg R, Wacker J. 1978. Asteroids as Geologic Materials: Collisions and Fragmentation" NASA Tech. Mem. 79729, 25-27

Cheng AF. 2002. Near Earth Asteroid Rendezvous: Mission summary. In Asteriods III (W. F. Bottke Jr. et al., eds.), Univ. of Arizona, Tucson.

Chesley SR, Farnocchia D, Nolan MC, Vokrouhlicky D, Chodas PW, et al. 2014. Orbit and bulk density of the OSIRIS-REx target Asteroid (101955) Bennu. Icarus 235:5-22

Chodas PW, Yeomans DK. 1996. The orbital motion and impact circumstances of Comet Shoemaker-Levy 9. In The Collision of Comet Shoemaker-Levy 9 and Jupiter (K. S. Noll et al., eds.), pp. 1-30. Proc. IAU Colloq. 156, Cambridge Univ., Cambridge.

Connolly HC, Lauretta DS, Walsh KJ, Tachibana S, Bottke WF. 2015. Towards understanding the dynamical evolution of asteroid 25143 Itokawa: constraints from sample analysis. Earth, Planets, and Space 67:12

Ćuk M, Burns JA. 2005. Effects of thermal radiation on the dynamics of binary NEAs. Icarus 176:418-32

Cuzzi JN, Hogan RC, Shariff K. 2008. Toward planetesimals: Dense chondrule clumps in the protoplanetary nebula. The Astrophysical Journal,687(2):1432-1447

Davis DR, Chapman CR, Weidenschilling SJ, Greenberg R. 1985. Collisional history of asteroids: Evidence from Vesta and the Hirayama families. Icarus 62:30-53

Davis DR, Weidenschilling SJ, Farinella P, Paolicchi P, Binzel RP. 1989. Asteroid collisional history: Effects on sizes and spins. In Asteroids II (R. P. Binzel et al., eds.), pp. 805- 826. Univ. of Arizona, Tucson.

Delbó M, dell'Oro A, Harris AW, Mottola S, Mueller M. 2007. Thermal inertia of near-Earth asteroids and implications for the magnitude of the Yarkovsky effect. Icarus 190:236-249

Delbó M, Libourel G, Wilkerson J, Murdoch N, Michel P, et al. 2014. Thermal fatigue as the origin of regolith on small asteroids. Nature 508:233-236

Delbo M, Mueller M, Emery JP, Rozitis B, Capria MT. 2015. Asteroid Thermophysical Modeling. in Asteroids IV 107-128 
Delbo M, Walsh K, Bolin B, Avdellidou C, Morbidelli A. 2017. Identification of a primordial asteroid family constrains the original planetesimal population Science 357:1026-1029

DeMeo FE, Carry B. 2013. The taxonomic distribution of asteroids from multi-filter all-sky photometric surveys. Icarus, 226:723-741.

DeMeo FE, Carry B. 2014. Solar System evolution from compositional mapping of the asteroid belt. Nature 505:629-634

DeMeo FE, Alexander CMOD, Walsh KJ, Chapman CR, Binzel RP. 2015. The Compositional Structure of the Asteroid Belt. Asteroids IV 13-41

Dobrovolskis AR. 1990. Tidal disruption of solid bodies. Icarus 88:24-38

Durda DD. 1996. The formation of asteroidal satellites in cata- strophic collisions. Icarus 120:212-219

Durda DD, Greenberg R, Jedicke R. 1998. Collisional models and scaling laws: A new interpretation of the shape of the main-belt asteroid size distribution. Icarus 135:431-440.

Durda DD, Bottke WF Jr, Enke BL, Merline WJ, Asphaug E, et al. 2004. The formation of asteroid satellites in large impacts: results from numerical simulations. Icarus 167:382-96

Durda DD, Bottke WF, Nesvorný D, Enke BL, Merline WJ, Asphaug E, Richardson, DC. 2007. Size-frequency distributions of fragments from SPH/N-body simulations of asteroid impacts: Comparison with observed asteroid families. Icarus 186(2):498-516

Durech J, Sidorin V, Kaasalainen M. 2010. DAMIT: a database of asteroid models. Astronomy and Astrophysics 513:A46

Durech J, Carry B, Delbo M, Kaasalainen M, Viikinkoski M. 2015. Asteroid Models from Multiple Data Sources. in Asteroids IV 183-202

Emery JP, Fernandez YR, Kelley MSP, Warden KT, Hergenrother C, et al. 2014. Thermal infrared observations and thermophysical characterization of OSIRIS-REx target asteroid (101955) Bennu. Icarus 234:17-35

Farinella P, Paolicchi P, Tedesco EF, Zappalà V. 1981. Triaxial equilibrium ellipsoids among the asteroids? Icarus, 46:114-123.

Fujiwara A, Kawaguchi J, Yeomans DK, Abe M, et al. 2006. The Rubble-Pile Asteroid Itokawa as Observed by Hayabusa. Science 312:1330-1334

Goldreich P, Sari R. 2009. Tidal evolution of rubble piles. The Astrophysical Journal 691(1):54-60

Gladman B, Michel P, Froeschle' C. 2000. The near-Earth object population. Icarus 146:176-89

Goldman DI, Umbanhowar, P. 2008. Scaling and dynamics of sphere and disk impact into granular media. Physical 
Review E 77(2):021308

Granvik M, Morbidelli A, Jedicke R, Bolin B, Bottke WF, et al. 2016. Super-catastrophic disruption of asteroids at small perihelion distances. Nature 530:303-306

Gundlach B, Blum J. 2013. A new method to determine the grain size of planetary regolith. Icarus 223:479-492

Guibout V, Scheeres DJ. 2003. Stability of Surface Motion on a Rotating Ellipsoid. CDMA 87:263-290.

Harris AW. 1998. A Thermal Model for Near-Earth Asteroids. Icarus 131:291-301

Harris AW, Fahnestock EG, Pravec P. 2009. On the shapes and spins of rubble pile asteroids. Icarus 199:310-318

Harris AW, Warner BD, Pravec P. 2016. Asteroid Lightcurve Derived Data V16.0. NASA Planetary Data System 246

Hirabayashi M, Scheeres DJ, Sánchez, DP, Gabriel T. 2014. Constraints on the Physical Properties of Main Belt Comet P/2013 R3 from its Breakup Event. The Astrophysical Journal 789:L12-L17

Hirabayashi M, Sánchez DP, Scheeres DJ. 2015. Internal Structure of Asteroids Having Surface Shedding Due to Rotational Instability. The Astrophysical Journal 808:63-75

Hirata N, Barnouin-Jha OS, Honda C, Nakamura R, Miyamoto H, et al. 2009. A survey of possible impact structures on 25143 Itokawa. Icarus 200:486-502

Holsapple KA. 1994. Catastrophic disruptions and cratering of Solar System bodies: A review and new results. Planetary and Space Science 42(12):1067-1078

Holsapple KA. 2001. Equilibrium Configurations of Solid Cohesionless Bodies. Icarus 154:432-448

Holsapple KA. 2004. Equilibrium figures of spinning bodies with self-gravity. Icarus 172:272-303

Holsapple KA, Michel P. 2006. Tidal disruptions: A continuum theory for solid bodies. Icarus 183:331-348

Holsapple KA, Michel P. 2008. Tidal disruptions. II. A continuum theory for solid bodies with strength, with applications to the Solar System. Icarus 193:283-301

Housen KR, Wilkening LL. 1982. Regoliths on small bodies in the solar system. Annual Review of Earth and Planetary Sciences 10(1):355-376

Housen KR, Holsapple KA. 1990. On the fragmentation of asteroids and planetary satellites. Icarus 84(1):226-253

Ishibashi K, Shirai K, Oqawa K, Wada K, Honda R, et al. 2017. Performance of Hayabusa2 DCAM3-D Camera for Short-Range Imaging of SCI and Ejecta Curtain Generated from the Artificial Impact Crater Formed on Asteroid 162137 Ryugu (1999 JU3). Space Science Reviews 208:213-238 
Jeffreys H. 1947. The relation of cohesion to Roche's limit. Mon. Not. R. Astron. Soc., 107:260-272

Johansen A, Jacquet E, Cuzzi JN, Morbidelli A, Gounelle M. 2015. New Paradigms for Asteroid Formation. in Asteroids IV 471-492

Johansen A, Oishi JS, Mac Low MM, Klahr H, Henning T, Youdin A, 2007. Rapid planetesimal formation in turbulent circumstellar disks. Nature 448(7157):1022-1026

Kaasalainen M, Ďurech J, Warner BD, Krugly YN, Gaftonyuk NM. 2007 Acceleration of the rotation of asteroid 1862 Apollo by radiation torques. Nature 446(7):420-422.

Lauretta DS, Balram-Knutson SS, Beshore E, Boynton,WV, d'Aubigny CD, et al. 2017. OSIRIS-REx: Sample Return from Asteroid (101955) Bennu. arXiv preprint arXiv:1702.06981

Lauretta DS, Bartels AE, Barucci MA, Bierhaus EB, Binzel RP, et al., 2015. The OSIRIS-REx target asteroid (101955) Bennu: Constraints on its physical, geological, and dynamical nature from astronomical observations. Meteoritics \& Planetary Science 50(4):834-849.

Margot J-L, Nolan MC, Benner LAM, Ostro SJ, Jurgens RF, et al. 2002. Binary asteroids in the near-Earth object population. Science 296:1445-48

Margot J-L, Pravec P, Taylor P, Carry B, Jacobson S. 2015. Asteroid Systems: Binaries, Triples, and Pairs. in Asteroids IV 355-374

Marshall J, Rizk B. 2015. Rounded boulders on Itokawa as clues to geological processes in the early solar system. Planetary and Space Science 119:181-184

Melosh HJ, Schenk P. 1993. Split comets and the origin of crater chains on Ganymede and Callisto. Nature, 365:731-733.

Melosh HJ, Stansberry JA. 1991. Doublet craters and the tidal disruption of binary asteroids. Icarus, 94:171-179.

Melosh HJ, Whitaker EA. 1994. Lunar crater chains. Nature, 369:713-714.

Michel P, Benz W, Tanga P, Richardson DC. 2001. Collisions and gravitational reaccumulation: Forming asteroid families and satellites. Science, 294:1696-1700

Michel P, Tanga P, Benz W, Richardson DC. 2002. Formation of asteroid families by catastrophic disruption:

Simulations with fragmentation and gravitational reaccumulation. Icarus 160(1):10-23

Michel P, Benz W, Richardson DC. 2003. Disruption of fragmented parent bodies as the origin of asteroid families. Nature 421(6923):608-612

Michel P, Benz W, Richardson DC. 2004. Catastrophic disruption of asteroids and family formation: a review of numerical simulations including both fragmentation and gravitational reaccumulations. Planet. Space Sci. 52:110917 
Michel P, O'Brien DP, Abe S, Hirata N. 2009. Itokawa's cratering record as observed by Hayabusa: Implications for its age and collisional history. Icarus 200:503-513

Michel P, Richardson DC. 2013. Collision and gravitational reaccumulation: Possible formation mechanism of the asteroid Itokawa. Astronomy \& Astrophysics 554:L1

Miyamoto H, Yano H, Scheeres D. J, et al. 2007. Regolith migration and sorting on asteroid Itokawa. Science 316(5827):1011-1014

Mottola S, Lahulla F. 2000. Mutual eclipse events in aster- oidal binary system 1996 FG3: Observations and a numeri- cal model. Icarus, 146:556-567.

Morbidelli A, Bottke WF, Nesvorný D, Levison HF. 2009. Asteroids were born big. Icarus 204(2):558-573.

Movshovitz N, Nimmo F, Korycansky DG, Asphaug E. Owen JM. 2016. Impact disruption of gravity-dominated bodies: New simulation data and scaling. Icarus 275:85-96

Mueller M, Harris AW, Fitzsimmons A. 2007. Size, albedo, and taxonomic type of potential spacecraft target Asteroid (10302) 1989 ML. Icarus 187:611-615

Müller TG, Hasegawa S, Usui F. 2014. (25143) Itokawa: The power of radiometric techniques for the interpretation of remote thermal observations in the light of the Hayabusa rendezvous results*. Publications of the Astronomical Society of Japan 66:52

Murdoch N, Sánchez P, Schwartz SR, Miyamoto H. 2015. Asteroid Surface Geophysics. in Asteroids IV 767-792

Nakamura A, Fujiwara A, 1991. Velocity distribution of fragments formed in a simulated collisional disruption. Icarus 92:132-146

Nesvorný D, Brož M, Carruba V. 2015. Identification and dynamical properties of asteroid families. Asteroids IV 297-321

Nolan MC, Magri C, Howell ES, Benner LAM, Giorgini JD, et al. 2013. Shape model and surface properties of the OSIRIS-REx target Asteroid (101955) Bennu from radar and lightcurve observations. Icarus 226:629-640

Öpik EJ. 1966. Sun-grazing comets and tidal disruption. Irish Astron. J. 7:141-161

Ostro SJ, Hudson RS, Benner LAM, Giorgini JD, Magri C, Margot J-L, Nolan MC. 2002. Asteroid radar astronomy. In Asteroids III (W. F. Bottke Jr. et al., eds.), this volume. Univ. of Arizona, Tucson.

Ostro SJ, Benner LAM, Nolan MC, Magri C, Giorgini JD, et al. 2004. Radar observations of asteroid 25143 Itokawa (1998 SF36). Meteoritics and Planetary Science 39:407-424

Ostro SJ, Margot JL, Benner LA, Giorgini JD, Scheeres DJ, et al. 2006. Radar imaging of binary near-Earth asteroid (66391) 1999 KW4. Science 314(5803):1276-1280

Pätzold M, Andert TP, Asmar SW, Anderson JD, Barriot JP, et al. 2011. Asteroid 21 Lutetia: Low mass, high density. Science 334(6055):491-492 
Pravec P, Hahn G. 1997. Two-period lightcurve of 1994 AW1: Indication of a binary asteroid? Icarus, 127:431-440.

Pravec P, Harris AW. 2000. Fast and slow rotation of asteroids. Icarus 148:12-20

Pravec P, Harris AW, Michalowski T. 2002. Asteroid rotations. See Bottke et al. 2002a, pp. 113-22

Pravec P, Scheirich P, Kušnirák P, Šarounová L, Mottola S, et al. 2006. Photometric survey of binary near-Earth asteroids. Icarus. 181(1):63-93

Richardson DC, Bottke WF. Jr, Love SG. 1998. Tidal distortion and disruption of Earth crossing asteroids. Icarus, 134:47-76.

Richardson DC, Quinn T, Stadel J, Lake G. 2000. Direct large-scale N-body simulations of planetesimal dynamics. Icarus, 143:45-59.

Richardson DC, Leinhardt ZM, Melosh HJ, Bottke WF Jr, Asphaug E. 2002. Gravitational aggregates: evidence and evolution. See Bottke et al. 2002a, pp. 501-15

Richardson DC, Elankumaran P, Sanderson RE. 2005. Numerical experiments with rubble piles: equilibrium shapes and spins. Icarus 173:349-61

Richardson DC, Walsh KJ. 2006. Binary Minor Planets. Annual Review of Earth and Planetary Sciences 34:47-81

Robinson MS, Thomas PC, Veverka J, Murchie SL, Wilcox BB. 2002. The geology of 433 Eros. Meteoritics and Planetary Science 37:1651-1684

Roche EA. 1847. Acad. Sci. Lett. Montpelier. Mem. Section Sci. 1:243

Rozitis B, Maclennan E, Emery JP. 2014. Cohesive forces prevent the rotational breakup of rubble-pile asteroid (29075) 1950 DA. Nature 512:174-176

Rubincam DP. 2000. Radiative spin-up and spin-down of small asteroids. Icarus 148:2-11

Sánchez P, Scheeres DJ. 2011. Simulating Asteroid Rubble Piles with A Self-gravitating Soft-sphere Distinct Element Method Model. The Astrophysical Journal 727:120-134

Sánchez P, Scheeres DJ. 2012. DEM simulation of rotation-induced reshaping and disruption of rubble-pile asteroids. Icarus 218:876-894

Sánchez P, Scheeres DJ. 2014. The strength of regolith and rubble pile asteroids. Meteoritics and Planetary Science 49:788-811

Sánchez P, Scheeres DJ. 2016. Disruption patterns of rotating self-gravitating aggregates: A survey on angle of friction and tensile strength. Icarus 271:453-471 
Saiki T, Imamura H, Arakawa M, Wada K, Takagi Y, et al. 2017. The Small Carry-on Impactor (SCI) and the Hayabusa2 Impact Experiment. Space Science Reviews 208:165-186

Saito J, Miyamoto H, Nakamura R, et al. 2006. Detailed images of asteroid 25143 Itokawa from Hayabusa. Science 312(5778):1341-1344

Scheeres DJ. 2015 Landslides and Mass shedding on spinning spheroidal asteroids. Icarus 247:1-17.

Scheeres DJ, Hesar SG, Tardivel S, Hirabayashi M, Farnocchia D, et al. 2016. The geophysical environment of Bennu. Icarus 276:116-140.

Scheirich P, Pravec P, Jacobson SA, Duurech J, Kusnirák P, et al. 2015. The binary near-Earth asteroid (175706) 1996 FG3 - An observational constraint on its orbital evolution. Icarus 245:56-63

Schenk PM, Asphaug E, McKinnon WB, Melosh HJ, Weissman PR. 1996. Cometary nuclei and tidal disruption: The geological record of crater chains on Callisto and Ganymede. Icarus, 121:249-274.

Schrapler R, Blum J, von Borstel I, Güttler C. 2015. The stratification of regolith on celestial objects. Icarus 257:3346

Schwartz SR, Richardson DC. Michel P. 2012. An implementation of the soft-sphere discrete element method in a high-performance parallel gravity tree-code. Granular Matter 14(3):363-380

Sharma I. 2009. The equilibrium of rubble-pile satellites: The Darwin and Roche ellipsoids for gravitationally held granular aggregates. Icarus 200:636-654

Slivan SM. 2002. Spin vector alignment of Koronis family asteroids. Nature 419(6):49-51

Solem JC. 1994. Density and size of comet Shoemaker-Levy 9 deduced from a tidal breakup model. Nature 370:349351

Solem JC, Hills JG. 1996. Shaping of Earth-crossing asteroids by tidal forces. Astron. J., 111:1382-1387.

Song C, Wang P, Makse HA. 2008. A phase diagram for jammed matter. Nature 453(7195):629-633

Sridhar S, Tremaine S. 1992. Tidal disruption of viscous bodies. Icarus, 95:86-99

Statler TS. 2009. Extreme sensitivity of the YORP effect to small- scale topography. Icarus 202(2):502-513

Sullivan RJ, Thomas PC, Murchie SL, Robinson MS. 2002. Asteroid Geology from Galileo and NEAR Shoemaker Data. in Asteroids III 331-350

Tanga P, Comito C, Paolicchi P, Hestroffer D, et al. 2009. Rubble-Pile Reshaping Reproduces Overall Asteroid Shapes. The Astrophysical Journal 706:L197-L202 
Taylor PA, Margot J.-L, Vokrouhlický D, Scheeres DJ, Pravec P, Lowry SC, Fitzsimmons A, Nolan MC, Ostro SJ, Benner LAM, Giorgini JD, Magri C. 2007. Spin rate of asteroid (54509) 2000 PH5 increasing due to the YORP effect. Science 316(5):274-277

Taylor PA, Howell ES, Nolan MC, Thane AA. 2012. The Shape and Spin Distributions of Near-Earth Asteroids Observed with the Arecibo Radar System. AAS/Division for Planetary Sciences Meeting Abstracts 44:302.07

Vokrouhlický D, Čapek D. 2002. YORP-induced long-term evolution of the spin state of small asteroids and meteoroids: Rubincam's approximation. Icarus 159:449-67

Vokrouhlický D, Nesvorný D, Bottke WF Jr. 2003. The vector alignments of asteroid spins by thermal torques. Nature 425:147-51

Vokrouhlický D, Brož M, Bottke WF, Nesvorný D, Morbidelli A. 2006. Yarkovsky/YORP chronology of asteroid families. Icarus 182(1):118-142

Walsh KJ, Richardson DC. 2006. Binary near-Earth asteroid formation: Rubble pile model of tidal disruptions. Icarus 180(1):201-216

Walsh KJ, Richardson DC. 2008. A steady-state model of NEA binaries formed by tidal disruption of gravitational aggregates. Icarus 193(2):553-566

Walsh KJ, Richardson DC, Michel P. 2008. Rotational breakup as the origin of small binary asteroids. Nature 454:188-191

Walsh KJ, Richardson DC, Michel P. 2012. Spin-up of rubble-pile asteroids: Disruption, satellite formation, and equilibrium shapes. Icarus 220:514-529

Walsh KJ. Jacobson SA, 2015. Formation and evolution of binary asteroids. in Asteroids IV 375-393

Walsh KJ, Ballouz RL, Durda DD, Richardson DC, Michel P, Jutzi M. 2017. Preserving Shape and Spin in Asteroid Reaccumulation Simulations with SSDEM. In Lunar and Planetary Science Conference (Vol. 48)

Weaver HA, Feldman PD, A’Hearn MF, Arpigny C, Brown RA, et al. 1994. Hubble Space Telescope Observations of Comet P/Shoemaker-Levy 9 (1993e). Science 263:787-791

Weidenschilling SJ. 1981. How fast can an asteroid spin? Icarus, 46:124-136.

Weissman PR. 1986. Are cometary nuclei primordial rubble piles? Nature 320:242-244 
Whiteley RJ, Tholen DJ, Hergenrother CW, 2002. Lightcurve Analysis of Four New Monolithic Fast-Rotating Asteroids. Icarus 157139-154

Wichman RW, Wood CA. 1995. The Davy crater chain: Implications for tidal disruption in the Earth-Moon system and elsewhere. Geophys. Res. Lett., 22:583-586.

Wurm G, Paraskov G, Krauss O. 2005. Growth of planetesimals by impacts at 25 m/s. Icarus 178(1):253-263.

Yasui M, Matsumoto E, Arakawa M. 2015. Experimental study on impact-induced seismic wave propagation through granular materials. Icarus 260:320-331

Yeomans DK, Chodas PW. 1994. The orbit of comet Shoemaker-Levy 9 about Jupiter. Bulletin of the American Astronomical Society 26:1022

Yeomans DK, Barriot JP, Dunham DW, Farquhar RW, Giorgini JD. et al. 1997. Estimating the mass of asteroid 253 Mathilde from tracking data during the NEAR flyby. Science 278(5346):2106-2109

Yeomans DK, 15 colleagues 2000. Radio science results during the NEAR-Shoemaker spacecraft rendezvous with Eros. Science, 289:2085-2088

Zhang Y. Richardson DC, Barnouin OS, Maurel C, Michel P. et al. 2017. Creep stability of the proposed AIDA mission target 65803 Didymos: I. Discrete cohesionless granular physics model. Icarus 294:98-123

Zhu, M-H, Fa W, Ip W-H, Huang J, Liu T, Meng L, Yan J, Xu A, Tang Z, Wang X, Qiao D. 2014. Morphology of asteroid (4179) Toutatis as imaged by Chang'E-2 spacecraft, Geophys. Res. Lett., 41:328-333

Zsom A, Ormel CW, Güttler C, Blum J. Dullemond CP. 2010. The outcome of protoplanetary dust growth: pebbles, boulders, or planetesimals?-II. Introducing the bouncing barrier. Astronomy \& Astrophysics, 513:A57

\section{REFERENCE ANNOTATION (15 word maximums):}

1. Asphaug \& Benz 1996 : This study used tidal breakup morphology to estimate SL9's density

2. Bottke et al. 1999: An amazing match between a tidal disruption simulation and radarobserved NEA.

3. Chapman et al. 1978: First use of the term "rubble pile" in the literature

4. Ćuk \& Burns 2005: First derivation of the Binary-YORP effect

5. DeMeo \& Carry 2014: Modern overview of asteroid physical and taxonomic distribution in the Main Belt and beyond 
6. Gundlach \& Blum 2013: Techniques to turn an observed thermal inertia into an average grain size.

7. Holsapple 2001: Important derivation of allowed shapes for cohesionless asteroids.

8. Margot et al. 2002: First radar discovered binary asteroid

9. Michel et al. 2001: End-to-end model of asteroid disruption, dispersal and reaccumulation of fragments

10. Yasui et al. 2015: Important study on seismic shaking

\section{SIDEBAR (200 words max -183 present):}

The YORP-effect relies on the asymmetries of an asteroid's shape to produce a torque around its spin axis when it reflects or re-emits solar radiation (Rubincam 2000, Bottke et al. 2006). The action of the YORP effect is directly observed in a handful of objects that are observed to be actively spinning up (Taylor et al. 2007, Kaasalainen et al. 2007) and long-term evolution is found in populations of asteroids that are driven into spin-orbit resonances in the Main Belt ("Slivan States" - Slivan et al. 2002; Vokrouhlický et al. 2003) and in the way that asteroid families drift apart over time (Vokrouhlický et al. 2006, Nesvorný et al. 2015). The YORP-effect can also alter obliquities, effectively pushing asteroids towards 0 or $180 \mathrm{deg}$ obliquities (Vokrouhlicky \& Čapek 2002,Čapek and Vokrouhlický 2004). Whether or not a specific asteroid is likely to be spinning up or spinning down is not-trivial to calculate, even for a well-studied body with a known shape, and some studies suggest that even small scale surface artifacts like boulders or very small craters can change how a body responds (Statler 2009).

\section{FIGURES:}

